\title{
ABSOLUTE CONTINUITY OF THE LAW FOR SOLUTIONS OF STOCHASTIC DIFFERENTIAL EQUATIONS WITH BOUNDARY NOISE
}

\author{
STEFANO BONACCORSI AND MARGHERITA ZANELLA
}

\begin{abstract}
We study existence and regularity of the density for the solution $u(t, x)$ (with fixed $t>0$ and $x \in D$ ) of the heat equation in a bounded domain $D \subset \mathbb{R}^{d}$ driven by a stochastic inhomogeneous Neumann boundary condition with stochastic term. The stochastic perturbation is given by a fractional Brownian motion process. Under suitable regularity assumptions on the coefficients, by means of tools from the Malliavin calculus, we prove that the law of the solution has a smooth density with respect to the Lebesgue measure in $\mathbb{R}$.
\end{abstract}

\section{INTRODUCTION}

Let $D$ be a bounded convex domain in $\mathbb{R}^{d}$ with smooth $C^{\infty}$ boundary $\partial D$, and denote $\nu$ the inwardpointing normal vector to $\partial D$. In the domain $D$, let us consider a heat equation with inhomogeneous boundary conditions of Neumann type

$$
\begin{cases}\partial_{t} u(t, x)-\frac{1}{2} \Delta u(t, x)=0, & t>0, x \in D \\ \langle\nu, \nabla u(t, \bar{x})\rangle+\beta u(t, \bar{x})+g(t, \bar{x})=0 & \bar{x} \in \partial D \\ u(0, x)=0 & x \in D\end{cases}
$$

where $g$ is given and $\beta$ is a positive constant.

Evolution problems of this kind have been studied by several authors; in particular, if $g$ were a deterministic function then equation (11) becomes a special case of the general theory of inhomogeneous boundary value problem, that is well studied in the literature, see for instance [11]. In this case, a solution for the heat problem is given by

$$
u(t, x)=\int_{0}^{t} \int_{\partial D} p_{N}(s, x, \bar{y}) g(s, \bar{y}) \mathrm{d} s,
$$

where $p_{N}(t, x, \bar{y})$ is the Poisson kernel of problem (11) (for further details see Section 2).

In our knowledge, the first extension to the case of stochastic boundary condition is due to [15] in case of an additive boundary white noise $g(t, \bar{x})=\alpha(\bar{x}) \frac{\mathrm{d}}{\mathrm{d} t} B(t)$ with respect to a Brownian motion process $B$. A semigroup approach to the same equation is given in [7]; the wave equation with stochastic boundary values was investigated in [12.

Stochastic perturbation driven by fractional Gaussian processes have been studied recently by several authors, see [9, 8, 13, 5. especially in the case of equations perturbed by distributed noise. Less results are available for the case of equations perturbed by a boundary noise: see [9, 4, 2, 6. In this last paper, authors allow also a nonlinear term on the boundary (both for the Brownian motion and the fractional Brownian motion).

2000 Mathematics Subject Classification. 60H15, $60 \mathrm{H} 07$.

Key words and phrases. Fractional Brownian motion, stochastic boundary condition, density of the solution. 
Here, we aim to investigate further the properties of the solution for the problem introduced in [1]. We consider the case when the boundary condition are given by the sum of a nonlinear function of the solution and a stochastic perturbation:

$$
g(t, \bar{x})=g(u(t, \bar{x}))-\int_{S} \alpha(\sigma, \bar{x}) \mathrm{d} B(\sigma, t),
$$

where $B(t, \sigma)$ is a fractional Brownian motion of Hurst parameter $H \in\left(\frac{1}{2}, 1\right)$ on $[0, T] \times S$ and we assume that $g: \mathbb{R} \rightarrow \mathbb{R}$ is a Lipschitz continuous function.

Thus we write the problem at hand in the form

$$
\begin{cases}\partial_{t} u(t, x)-\frac{1}{2} \Delta u(t, x)=0, & t>0, x \in D, \\ \langle\nu, \nabla u(t, \bar{x})\rangle+\beta u(t, \bar{x})+g(u(t, \bar{x}))=\frac{\mathrm{d}}{\mathrm{d} t} \int_{S} \alpha(\sigma, \bar{x}) \mathrm{d} B(\sigma, t), & \bar{x} \in \partial D, \\ u(0, x)=0 & x \in D .\end{cases}
$$

First, we prove the existence, uniqueness and continuity of the solution $u(t, x)$ with respect to both parameters $t$ and $x$. Further, we show, using tools of the Malliavin calculus, that the random variable $u(t, x)$ has an absolutely continuous probability law and, under suitable assumptions, the density of this random variable is smooth.

We interpret equation (3) in the sense of Walsh [16]: a solution of (3) is the process $u(t, x)$ that satisfies the evolution equation

$$
u(t, x)=\int_{0}^{t} \int_{\partial D} p_{N}(t-s, x, \bar{y}) g(u(s, \bar{y})) \mathrm{d} \bar{y} \mathrm{~d} s+\int_{0}^{t} \int_{S} \int_{\partial D} p_{N}(t-s, x, \bar{y}) \alpha(\sigma, \bar{y}) \mathrm{d} \bar{y} \mathrm{~d} B(\sigma, s) .
$$

In order to give a sense to the random field formulation for the solution we have to evaluate the process in both time and space. It is then necessary to prove that there exists a continuous version (in both the entries) of the process. As regards the continuity in time, it is a classical result and it follows immediately from (4). As regards the space regularity, in the following we prove that we can consider a continuous version of the solution process inside the domain $D$; unfortunately this kind of regularity can not be extended to the boundary $\partial D$ as well, where we have only a regularity of $L^{p}(\partial D)$ type. Our main aim is to prove the existence of a smooth density for the solution at time $t$ and space $x \in D$ fixed. In order to obtain this kind of result we need, as a necessary step, to study the solution process, as well as its Malliavin derivative, on the boundary. We point out here that, since we do not have the continuity in space on the boundary, all the results stated for $u(t, \xi)$ with $\xi \in \partial D$ (and for its Malliavin derivative) are valid a.e. and, with an abuse of notation, by $u(t, \xi)$ we mean a representative element in the equivalence class $L^{p}(\partial D)$. Nevertheless, as regards the analogous results stated for the points inside the domain, they are valid everywhere thanks to the representation formula for the solution and its Malliavin derivative (see (22) and (27)).

Let us state the main assumptions that we impose on the coefficients of the problem.

(g1): Lipschitz continuity. We assume that $g \in C^{1}(\mathbb{R})$ is Lipschitz continuous, with $\left|\partial_{u} g(u)\right| \leq L$ and $g(u) \leq L(1+|u|)$ for some constant $L>0$.

(g2): Smoothness. Further to (g1), in order to prove the smoothness of the law of the solution $u(t, x)$ we shall require that $g \in C^{\infty}(\mathbb{R})$ and derivatives of all orders are bounded by a (finite) constant $L:\left\|\partial_{u}^{n} g(u)\right\| \leq L$ for every $n \geq 1$.

(a1): Integrability of the boundary coefficient. We assume that $\alpha \in L^{2}(S \times \partial D)$.

(a1'): Further integrability of the boundary coefficient. In addition to (a1), for some results we will require a stronger integrability condition of the boundary coefficient, namely that $\alpha \in L^{\theta}\left(\partial D ; L^{2}(S)\right)$ for $\theta>\frac{d-1}{2 H-1}$. 
(a2): Non-degeneracy. There exists $\alpha_{0}>0$ such that $\alpha(\sigma, x) \geq \alpha_{0}$.

This paper is in principle an extension of the results in [1, 2, 6]. Section 2 contains some preliminaries, basic definitions and construction of the heat kernel in a bounded domain. Although these results should be classical, we have find it useful to collect them in a unique place; moreover, we did not find a reference for some of the estimates we need here.

Section 3 contains a review of some basic facts about Malliavin calculus for fractional Gaussian processes (our approach here is based mainly on [14]).

Section 4 is devoted to the analysis of the stochastic convolution process: we first study the regularity of the process and then we compute its Malliavin derivative. We also prove that its law admits a density with respect to the Lebesgue measure.

In Section 5 we recall the results concerning the existence and regularity of the solution of (3) proved in [1] and we study the Malliavin derivative of the solution. Finally in Sections 6 we study the regularity of the solution in the Malliavin sense.

Notation. In the sequel, we shall indicate with $C$ a constant that may vary from line to line. In certain cases, we write $C_{\alpha, \beta, \ldots}$ to emphasize the dependence of the constant on the parameters $\alpha, \beta, \ldots$.

\section{Some useful estimates on the Poisson Kernel}

In this section we review the construction of the fundamental solution of the heat equation with inhomogeneous boundary conditions and we state the relevant estimates that we need in the sequel. There is a large literature concerning this subject: we shall refer, for instance, to [10, Chapter 5].

Let $D \subset \mathbb{R}^{d}$ be a bounded domain, with smooth boundary $\partial D$. We denote $\sigma(\mathrm{d} y)$ the surface measure on $\partial D$ and we assume that $|\partial D|<\infty$.

Let us consider the problem

$$
\begin{cases}\frac{\partial}{\partial t} u(t, x)-\frac{1}{2} \Delta u(t, x)=0 & t \in(0, T), \quad x \in \stackrel{\circ}{ } \\ u(0, x)=0 & x \in D \\ \frac{\partial}{\partial \nu} u(t, \xi)+\beta u(t, \xi)=g(t, \xi) & t \in(0, T), \quad \xi \in \partial D\end{cases}
$$

Set $L=\frac{1}{2} \Delta-\frac{\partial}{\partial t}$. We extend the coefficients of $L$ to a larger set $\Omega_{0}:=D_{0} \times[0, T]$, where $D_{0}$ is a bounded domain containing $\bar{D}$ (notice that the extension is trivial since the coefficients are constant). The fundamental solution $\Gamma(t, x, s, y)$ can be constructed in $\Omega_{0}$. Let us recall that

Definition 1. A fundamental solution of $L u=0$ in $\Omega_{0}$ is a function $\Gamma$ defined for all $(t, x) \in \Omega_{0}$, $(s, y) \in \Omega_{0}, t>s$, which satisfies the following conditions:

(1) for fixed $(s, y) \in \Omega_{0}$, it satisfies the equation $L u=0$;

(2) for any continuous function $f(x) \in C\left(\bar{D}_{0}\right)$, if $x \in D_{0}$ then

$$
\lim _{t \downarrow s} \int_{D_{0}} \Gamma(t, x, s, y) f(y) \mathrm{d} y=f(x) .
$$

According to [10, Chapter 5.1], the fundamental solution of the homogeneous problem associated to (5) is given by a function $Z(t, x, s, y)$, that is the heat kernel for the $d$-dimensional Brownian motion associated with the diffusion operator $L$, so it can be written as

$$
Z(t, x, s, y)=(2 \pi(t-s))^{-\frac{d}{2}} \exp \left(-\frac{|x-y|^{2}}{2 t}\right) .
$$


According to this representation, the following inequalities hold for $x, y \in D_{0}$ and $0 \leq s<t \leq T$ :

$$
\begin{aligned}
|\Gamma(t, x, s, y)| & \leq c(t-s)^{-\mu}|x-y|^{2 \mu-d}, \quad 0<\mu<1 ; \\
\left|\nabla_{x} \Gamma(t, x, s, y)\right| & \leq c(t-s)^{-\mu}|x-y|^{2 \mu-d-1}, \quad \frac{1}{2}<\mu<1 .
\end{aligned}
$$

Let us now return to problem (5). The following result (see [10, Theorem 5.3.2]) provides an explicit formula for the associated kernel and a first useful estimate.

Theorem 2. If $g$ is a continuous function on $\partial D \times[0, T]$, then there exists a unique solution of the problem (5), and it has the form

$$
u(t, x)=\int_{0}^{t} \int_{\partial D} p_{N}(t, x, s, y) g(s, y) \sigma(\mathrm{d} y) \mathrm{d} s,
$$

where

$$
p_{N}(t, x, s, y)=-2 \Gamma(t, x, s, y)-2 \sum_{n=1}^{\infty} \int_{s}^{t} \int_{\partial D} \Gamma(t, x, s, y) M_{n}(r, z, s, y) \sigma(\mathrm{d} z) \mathrm{d} r
$$

and the terms $M_{n}$ are defined recursively as follows

$$
\begin{aligned}
M_{1}(t, x, s, y) & :=M(t, x, s, y)=\left[\frac{\partial \Gamma}{\partial \nu(x)}+\beta \Gamma\right](t, x, s, y) \\
M_{n+1} & :=\int_{0}^{t} \int_{\partial D} M(t, x, r, z) M_{n}(r, z, s, y) \sigma(\mathrm{d} y) \mathrm{d} s .
\end{aligned}
$$

We shall not provide the proof of the theorem; however, we concentrate on the following consequence of the construction. Some technical lemmas, that are needed in order to get the proof, are given in the Appendix.

Corollary 3. For every $(t, x) \in[0, T] \times \bar{D}$, the following estimate holds

$$
p_{N}(t, x, s, y) \leq c(t-s)^{-\mu}|x-y|^{2 \mu-d}, \quad \frac{1}{2}<\mu<1 .
$$

Proof. We start from (77); using Lemma 26] we have

$$
|M(t, x, s, y)| \leq c(t-s)^{-\mu}|x-y|^{2 \mu-d} .
$$

So, proceeding by induction, and using Lemma 25, we can give an explicit estimate for all the $M_{n}$ 's. For $M_{1}$ we have the estimate above. Let us explicitly show the case $M_{2}$.

$$
\begin{aligned}
\left|M_{2}(t, x, s, y)\right| & \leq c^{2} \int_{s}^{t} \int_{\partial D}(t-r)^{-\mu}|x-z|^{2 \mu-d}(r-s)^{-\mu}|z-y|^{2 \mu-d} \sigma(\mathrm{d} z) \mathrm{d} r \\
& =c^{2}\left(\int_{s}^{t}(t-r)^{-\mu}(r-s)^{-\mu} \mathrm{d} r\right)|x-y|^{4 \mu-d-1} \\
& \leq c^{2} \frac{\Gamma(1-\mu) \Gamma(1-\mu)}{\Gamma(2-2 \mu)}(t-s)^{1-2 \mu}|x-y|^{4 \mu-d-1}
\end{aligned}
$$

Then the induction step allows us to conclude

$$
\left|M_{k}(t, x, s, y)\right| \leq \gamma_{k}(\mu) c^{k}(t-s)^{k(1-\mu)-1}|x-y|^{2 k \mu-d-k+1}
$$


where

$$
\gamma_{k}(\mu)=\prod_{j=1}^{k} C_{j}(\mu)
$$

with

$$
C_{j}(\mu)=\frac{\Gamma(1-\mu) \Gamma(j(1-\mu)}{\Gamma((j+1)(1-\mu))} .
$$

It follows immediately that

$$
\left|M_{k}(t, x, s, y)\right| \leq c^{k} \frac{(\Gamma(1-\mu))^{k+1}}{\Gamma((k+1)(1-\mu))}(t-s)^{k(1-\mu)-1}|x-y|^{k(2 \mu-1)+1-d} .
$$

Using previous estimate on the $M_{k}$ 's and (77), we can now compute the desired estimate of the kernel, starting from expression (10). Let us start by estimating an arbitrary term of the series in (10) (we need again Lemma 25):

$$
\begin{aligned}
\int_{s}^{t} \int_{\partial D} & \Gamma(t, x, s, y) M_{n}(r, z, s, y) \sigma(\mathrm{d} z) \mathrm{d} r \\
& \leq c^{k} \frac{(\Gamma(1-\mu))^{k+1}}{\Gamma((k+1)(1-\mu))} \int_{s}^{t} \int_{\partial D}(t-r)^{-\mu}|x-y|^{2 \mu-d}(r-s)^{k(1-\mu)-1}|y-z|^{k(2 \mu-1)+1-d} \sigma(\mathrm{d} z) \mathrm{d} r \\
& \leq c^{k} \frac{(\Gamma(1-\mu))^{k+1}}{\Gamma((k+1)(1-\mu))} \frac{\Gamma(1-\mu) \Gamma(k(1-\mu))}{\Gamma((k+1)(1-\mu))}(t-s)^{k(1-\mu)-\mu} C|x-y|^{k(2 \mu-1)+2 \mu-d}
\end{aligned}
$$

and so we get

$$
\begin{aligned}
& \left|p_{N}(t, x, s y)\right| \leq c(t-s)^{-\mu}|x-y|^{2 \mu-d} \\
& \quad+\left(c \sum_{k=1}^{\infty} \frac{c^{k+1}(\Gamma(1-\mu))^{k+1}}{\Gamma((k+1)(1-\mu))}(t-s)^{k(1-\mu)}|x-y|^{k(2 \mu-1)}\right)|x-y|^{2 \mu-d}(t-s)^{-\mu},
\end{aligned}
$$

where the series is convergent provided $\frac{1}{2}<\mu<1$. So we recover the desired estimate

$$
p_{N}(t, x, s, y) \leq c(t-s)^{-\mu}|x-y|^{2 \mu-d} \text {. }
$$

Remark 1. Notice that the estimate made on the kernel $p_{N}(t, x, s, y)$ is valid also when $x \in \partial D$ thanks to Lemma 26 and the fact that formula (7) is true for all $x \in D_{0} \supset \bar{D}$.

We state now a lower bound estimates for the Poisson kernel: we refer to [17, Theorem 4.1].

Lemma 4. Suppose that domain $D$ is convex. There exist constants $C_{1}, C_{2}>0$ and $T>0$, such that for $x, y \in D, t \in[0, T]$

$$
p_{N}(t, x, y) \geq C_{1} t^{-\frac{d}{2}} \exp \left(-\frac{C_{2}|x-y|^{2}}{t}\right) .
$$

Finally we give some useful estimates about the spatial derivative of the kernel. The following lemma is provided in [15, Proposition 3.3]

Lemma 5. There exists a constant $k \leq 1$ such that

$$
\left|\nabla p_{N}(t, x, \bar{y})\right| \leq k^{-1} \exp \left(-k|x-\bar{y}|^{2} / t\right) t^{-(d+1) / 2}
$$

for all $t \in(0, T), x \in \stackrel{\circ}{D}$ and $\bar{y} \in \partial D$. 
The above estimate is not so easy to deal with, because of the presence of the Gaussian exponential. However, if we recall the inequalities (43) and (44), i.e.,

$$
t^{-n / 2} e^{-k r^{2} / t} \leq K t^{-\mu} r^{-n+2 \mu}
$$

then we have the following.

Corollary 6. There exists a constant $k>0$ such that

$$
\left|\nabla p_{N}(t, x, \bar{y})\right| \leq k|x-\bar{y}|^{-d+2 \mu} t^{-(2 \mu+1) / 2}
$$

for all $t \in(0, T), x \in \stackrel{\circ}{D}$ and $\bar{y} \in \partial D$.

\section{Preliminaries on Malliavin calculus}

Let us recall some basic facts about the Malliavin calculus with respect to (standard and fractional) Brownian motion; for full details, we refer to [14].

Fix a measurable space $(S, \mathcal{S})$ with a finite measure $\mu$ on it, as well as a time interval $[0, T]$.

We are given a complete probability space $(\Omega, \mathcal{F}, \mathbb{P})$ and a centered Gaussian family $B=\{B(h), h \in$ $\mathcal{H}$ \} defined in $\Omega$. The space $\mathcal{H}$ is constructed below.

Recall that a fractional Brownian motion $B^{H}=\left\{B^{H}(t), t \in[0, T]\right\}$ is a centered Gaussian process with covariance function

$$
R_{H}(t, s)=\frac{1}{2}\left(s^{2 H}+t^{2 H}-|t-s|^{2 H}\right), \quad s, t \in[0, T] .
$$

Let $\mathcal{E}$ be the space of step functions on $[0, T] \times S$. We denote by $\mathcal{H}$ the closure of $\mathcal{E}$ with respect to the scalar product

$$
\left\langle\mathbb{1}_{[0, t]} \times \mathbb{1}_{A}, \mathbb{1}_{[0, s]} \times \mathbb{1}_{B}\right\rangle_{\mathcal{H}}=R_{H}(t, s) \mu(A \cap B) ;
$$

notice that in case $H=1 / 2$ then the first component in $\mathcal{H}$ is the standard $L^{2}$ space with respect to the Lebesgue measure on $[0, T]$, so that for $\phi, \psi \in \mathcal{H}$ we have

$$
\langle\phi, \psi\rangle_{\mathcal{H}}=\int_{0}^{T} \int_{S} \phi(s, \sigma) \psi(s, \sigma) \mu(\mathrm{d} \sigma) \mathrm{d} s .
$$

In case of a fractional Brownian motion with Hurst parameter $H>1 / 2$ it holds

$$
\langle\phi, \psi\rangle_{\mathcal{H}}=\int_{S} \int_{0}^{T} \int_{0}^{T}|s-t|^{2 H-2} \phi(s, \sigma) \psi(t, \sigma) \mathrm{d} t \mathrm{~d} s \mu(\mathrm{d} \sigma),
$$

$\phi, \psi \in \mathcal{H}$.

Thus, in case $H=1 / 2$, we say that the Gaussian family $B$ is associated to a Brownian motion process $B_{s, \sigma}$ on $\mathcal{H}$ and in case $H>\frac{1}{2}$ it is associated to a fractional Brownian motion $B_{s, \sigma}$ via the identification

$$
B(\phi)=\int_{0}^{T} \int_{S} \phi(s, \sigma) \mathrm{d} B_{s, \sigma}, \quad \phi \in \mathcal{H} .
$$

A $\mathcal{F}$-measurable real valued random variable $F$ is said to be cylindrical if it can be written as

$$
F=f\left(B\left(\phi^{1}\right), \ldots, B\left(\phi^{n}\right)\right),
$$


where $\phi^{i} \in \mathcal{H}$ and $f: \mathbb{R}^{n} \rightarrow \mathbb{R}$ is a $C^{\infty}$ bounded function. The set of cylindrical random variables is denoted $\mathcal{S}$. The Malliavin derivative of $F \in \mathcal{S}$ is the stochastic process $D F=\left\{D_{s, \sigma} F, s \in[0, T], \sigma \in\right.$ $S$ \} given by

$$
D_{s, \sigma} F=\sum_{i=1}^{n} \phi^{i}(s, \sigma) \frac{\partial f}{\partial x_{i}}\left(B\left(\phi^{1}\right), \ldots, B\left(\phi^{n}\right)\right) .
$$

More generally, we can introduce iterated derivatives. If $F \in \mathcal{S}$, we set

$$
D_{t_{1}, \ldots, t_{k}, \sigma_{1}, \ldots, \sigma_{k}}^{k} F=D_{t_{1}, \sigma_{1}} \ldots D_{t_{k}, \sigma_{k}} F \text {. }
$$

For any $p \geq 1$, the operator $D^{k}$ is closable from $\mathcal{S}$ into $L^{p}\left(\mathcal{C}([0, T] \times S, \mathbb{R}), \mathcal{H}^{\otimes k}\right)$. We denote by $\mathbb{D}^{k, p}(\mathcal{H})$ the closure of the class of cylindrical random variables with respect to the norm

$$
\|F\|_{k, p}=\left(\mathbb{E}\left(|F|^{p}\right)+\sum_{j=1}^{k} \mathbb{E}\left(\left\|D^{j} F\right\|_{\mathcal{H} \otimes j}^{p}\right)\right)^{\frac{1}{p}}
$$

and

$$
\mathbb{D}^{\infty}(\mathcal{H})=\bigcap_{p \geq 1} \bigcap_{k \geq 1} \mathbb{D}^{k, p}(\mathcal{H})
$$

We also introduce the localized spaces $\mathbb{D}_{\text {loc }}^{k, p}(\mathcal{H})$ by saying that a random variable $F$ belongs to $\mathbb{D}_{\text {loc }}^{k, p}(\mathcal{H})$ if there exists a sequence of sets $\Omega_{n} \subset \Omega$ and random variables $F_{n} \in \mathbb{D}^{k, p}(\mathcal{H})$ such that $\Omega_{n} \uparrow \Omega$ almost surely and such that $F=F_{n}$ on $\Omega_{n}$.

We then have the following key result which stems from Theorem 2.1.2 and Corollary 2.1.2. in [14]:

Theorem 7. Let $F=\left(F_{1}, \ldots, F_{n}\right)$ be a $\mathcal{F}$-measurable random vector such that:

(1) For every $i=1, \ldots, n, F_{i} \in \mathbb{D}_{\text {loc }}^{1,2}(\mathcal{H})$;

(2) The Malliavin matrix of the random vector $F: \Gamma=\left(\left\langle D F^{i}, D F^{j}\right\rangle_{\mathcal{H}}\right)_{1 \leq i, j \leq n}$ is invertible almost surely.

Then the law of $F$ has a density with respect to the Lebesgue measure on $\mathbb{R}^{n}$.

If moreover $F \in \mathbb{D}^{\infty}(\mathcal{H})$ and, for every $p>1$,

$$
\mathbb{E}\left(|\operatorname{det} \Gamma|^{-p}\right)<+\infty,
$$

then this density is smooth.

The following result is useful in the proof of regularity for the solution of the stochastic differential equation (3). We recall here for the sake of completeness. A proof can be found, for instance, in [14. Lemma 1.5.3].

Proposition 8. Let $\left\{F_{n}\right\}$ be a sequence of variables in $\mathbb{D}^{k, p}$ for some $p>1$. Assume that the sequence $F_{n}$ converges to $F$ in $L^{p}(\Omega)$ and that

$$
\sup _{n}\left\|F_{n}\right\|_{k, p}<\infty
$$

Then $F$ belongs to $\mathbb{D}^{k, p}$. 


\section{The Stochastic CONVOLUtion PROCESS}

We consider first the stochastic convolution term

$$
Z(t, x)=\int_{0}^{t} \int_{S} \int_{\partial D} p_{N}(t-s, x, \bar{y}) \alpha(\sigma, \bar{y}) \mathrm{d} \bar{y} \mathrm{~d} B(\sigma, s)
$$

from equation (4). For the sake of completeness we recall some basic facts about Wiener integral with respect to fractional Brownian motion. For more details see, for instance [3]. In Section 3 we have introduced the fractional Brownian motion as a Gaussian process with covariance function given by (17). It is useful having in mind another characterization of the process which will allows us to do all the required computations.

A fractional Brownian motion $B(\sigma, s)$ of Hurst parameter $H$ can be defined as the convolution product

$$
B(\sigma, t)=\int_{0}^{t} \int_{S} K_{H}(t-s) \mathrm{d} W(\sigma, s)
$$

where $\{W(\sigma, t), \sigma \in S, t \in[0, T]\}$ is a standard Brownian motion and $K_{H}$ is the kernel

$$
K_{H}(t, s)=C_{H}(t-s)^{H-\frac{1}{2}}+C_{H}\left(\frac{1}{2}-H\right) \int_{s}^{t}(u-s)^{H-\frac{3}{2}}\left(1-\left(\frac{s}{u}\right)^{\frac{1}{2}-H}\right) \mathrm{d} u .
$$

where $C_{H}$ is a normalizing constant, given by

$$
C_{H}=\left(\frac{2 H \Gamma\left(\frac{3}{2}-H\right)}{\Gamma\left(H+\frac{1}{2}\right) \Gamma(2-2 H)}\right)^{\frac{1}{2}}
$$

Let us consider the integral

then the Itô isometry reads as

$$
\mathcal{I}(\varphi)=\int_{0}^{t} \int_{S} \varphi(\sigma, r) \mathrm{d} B(\sigma, r)
$$

$$
\mathbb{E}|\mathcal{I}(\varphi)|^{2}=\int_{0}^{t} \int_{S}\left|K^{*}(\varphi(\sigma, \cdot))(s)\right|^{2} \mu(\mathrm{d} \sigma) \mathrm{d} s
$$

for an operator $K^{*}$ which maps the reproducing kernel Hilbert space $\mathcal{H}$ into $L^{2}(0, T)$ :

$$
\left(K^{*} \varphi\right)(s)=\mathbf{1}_{(0, t)}(s) \int_{s}^{t} \varphi(\cdot, r)\left(\frac{s}{r}\right)^{\frac{1}{2}-H}(r-s)^{H-\frac{3}{2}} \mathrm{~d} r .
$$

4.1. Global regularity and smoothness in space of the convolution process. Next proposition is related to space regularity of the process $Z(t, x)$.

Proposition 9. For any $t \in[0, T]$, there exists a version of the stochastic convolution process

$$
Z(t, x)=\int_{0}^{t} \int_{S} \int_{\partial D} p_{N}(t-s, x, \bar{y}) \alpha(\sigma, \bar{y}) \mathrm{d} \bar{y} \mathrm{~d} B(\sigma, s)
$$

that is a continuous function on $D$ and locally Hölder continuous, of arbitrary exponent $\gamma<1$.

Proof. The thesis follows from Kolmogorov's criterium of continuity and the Gaussian character of the stochastic convolution process. Let for simplicity be $\phi(t, x, \sigma)=\int_{\partial D} p_{N}(t, x, \bar{y}) \alpha(\sigma, \bar{y}) \mathrm{d} \bar{y}$; first, we have that

$$
\mathbb{E}|Z(t, x)|^{2}=\int_{0}^{t} \int_{S}\left|\left(K^{*}\left(\phi(t-\cdot, x, \sigma) 1_{(0, t)}(\cdot)\right)\right)(s)\right|^{2} \mu(\mathrm{d} \sigma) \mathrm{d} s
$$


Fix $x \in D$ and $M$ a convex neighbourhood of $x$ strictly contained in $D, \operatorname{dist}(M, \partial D)>\varepsilon$. For any $z \in M$ it follows

$$
\begin{aligned}
\mathbb{E} & |Z(t, x)-Z(t, z)|^{2}=\int_{0}^{t} \int_{S}\left|\left(K^{*}\left([\phi(t-\cdot, x, \sigma)-\phi(t-\cdot, z, \sigma)] 1_{(0, t)}(\cdot)\right)\right)(s)\right|^{2} \mu(\mathrm{d} \sigma) \mathrm{d} s \\
= & \int_{0}^{t} \int_{S}\left|\int_{s}^{t}\left(\frac{s}{r}\right)^{1 / 2-H}(r-s)^{H-3 / 2}[\phi(t-r, x, \sigma)-\phi(t-r, z, \sigma)] \mathrm{d} r\right|^{2} \mu(\mathrm{d} \sigma) \mathrm{d} s \\
\leq & t^{2 H-1} \int_{0}^{t} \int_{S} s^{1-2 H}\left|\int_{s}^{t}(r-s)^{H-3 / 2}[\phi(t-r, x, \sigma)-\phi(t-r, z, \sigma)] \mathrm{d} r\right|^{2} \mu(\mathrm{d} \sigma) \mathrm{d} s
\end{aligned}
$$

We may estimate, by using Corollary 6 for some $\mu \in\left(\frac{1}{2}, H\right)$ (that is always possible, since $H>\frac{1}{2}$ by assumption)

$$
\begin{aligned}
\mid \phi(t-r, x, \sigma)- & \phi(t-r, z, \sigma)|=| \int_{\partial D}\left[p_{N}(t-r, x, \bar{y})-p_{N}(t-r, z, \bar{y})\right] \alpha(\sigma, \bar{y}) \mathrm{d} \bar{y} \mid \\
& \leq \int_{\partial D} \int_{0}^{1}\left|\frac{\partial}{\partial u} p_{N}(t-r, x+u(z-x), \bar{y})\right| \mathrm{d} u \alpha(\sigma, \bar{y}) \mathrm{d} \bar{y} \\
& \leq|z-x| \int_{\partial D} \int_{0}^{1}\left|\nabla p_{N}(t-r, x+u(z-x), \bar{y})\right| \mathrm{d} u \alpha(\sigma, \bar{y}) \mathrm{d} \bar{y} \\
& \leq(t-r)^{-\mu}|z-x| \int_{\partial D}\left(\int_{0}^{1}|x+u(z-x)-\bar{y}|^{-(d+1)+2 \mu} \mathrm{d} u\right) \alpha(\sigma, \bar{y}) \mathrm{d} \bar{y} \\
& \leq(t-r)^{-\mu}|z-x| \varepsilon^{-(d+1)+2 \mu} \int_{\partial D} \alpha(\sigma, \bar{y}) \mathrm{d} \bar{y}
\end{aligned}
$$

where we recall that $\varepsilon>0$ is the distance between $M$ and $\partial D$. Thus we obtain

$$
\begin{aligned}
& \mathbb{E}|Z(t, x)-Z(t, z)|^{2} \\
& \leq t^{2 H-1} \int_{0}^{t} \int_{S} s^{1-2 H}\left|\int_{s}^{t}(r-s)^{H-3 / 2}\left[(t-r)^{-\mu}|z-x| \varepsilon^{-(d+1)+2 \mu} \int_{\partial D} \alpha(\sigma, \bar{y}) \mathrm{d} \bar{y}\right] \mathrm{d} r\right|^{2} \mu(\mathrm{d} \sigma) \mathrm{d} s \\
& \leq|z-x|^{2} \varepsilon^{-2(d+1)+4 \mu}\|\alpha\|_{L^{2}(\partial D \times S)}^{2} t^{2 H-1} \int_{0}^{t} s^{1-2 H}\left|\int_{s}^{t}(r-s)^{H-3 / 2}(t-r)^{-\mu} \mathrm{d} r\right|^{2} \mathrm{~d} s
\end{aligned}
$$

an explicit computation for the inner integral leads to

$$
\mathbb{E}|Z(t, x)-Z(t, z)|^{2} \leq C_{H, \mu}^{2}|z-x|^{2} \varepsilon^{-2(d+1)+4 \mu}\|\alpha\|_{L^{2}(\partial D \times S)}^{2} t^{2 H-1} \int_{0}^{t} s^{1-2 H}(t-s)^{2 H-2 \mu-1} \mathrm{~d} s
$$

hence

$$
\mathbb{E}|Z(t, x)-Z(t, z)|^{2} \leq C_{H, \mu, \alpha, \varepsilon} t^{2 H-2 \mu}|z-x|^{2} .
$$

Taking into account that $Z(t, x)-Z(t, z)$ is a centered Gaussian random variable with variance bounded by the right-hand side of previous inequality, we have for any $k \in \mathbb{N}$

$$
\mathbb{E}|Z(t, x)-Z(t, z)|^{2 k} \leq C_{k, T, H, \mu, \alpha, \varepsilon}|z-x|^{2 k} .
$$

For $k$ large enough, writing $2 k=d+(2 k-d)$, we appeal to Kolmogorov's continuity theorem for random fields to conclude that there exists a modification of $Z(t, \cdot)$ that is continuous on $M$ and $\gamma$-Hölder continuous for arbitrary $\gamma<\frac{2 k-d}{2 k}$ hence, sending $k$ to infinity, for arbitrary $\gamma<1$. 
Remark 2. Under condition (a1) it can be easily shown that, for every $(t, x) \in[0, T] \times D, Z(t, x)$ is an $L^{p}(\Omega)$ random variable, for every $p \geq 2$. The proof follows by using almost the same computations used in the proof of Proposition 9.

In order to give a meaning to the nonlinear boundary conditions, we shall prove that this process has a trace on the boundary $\partial D$, compare [1, Lemma 2.7].

Definition 10. If $E$ is a separable Banach space, we denote by $L_{W}^{q}(0, T ; E)$ the space $L^{q}\left(0, T ; L^{q}(\Omega, \mathcal{F}, \mathbb{P} ; E)\right)$ of all $\left\{\mathcal{F}_{t}\right\}_{t \geq 0}$-adapted mappings $X:(0, T) \rightarrow L^{q}(\Omega, \mathcal{F}, \mathbb{P} ; E)$ with the norm

$$
\|X\|_{L_{W}^{q}(0, T ; E)}=\left(\int_{0}^{T} \mathbb{E}|X(t)|_{E}^{q} \mathrm{~d} t\right)^{\frac{1}{q}}
$$

where $\mathbb{E}$ is the expectation in the probability space $(\Omega, \mathcal{F}, \mathbb{P})$.

Lemma 11. Let us assume the non degeneracy hypothesis (a1'), then the boundary trace of the stochastic convolution process $Z(t, \xi)$ belongs to $L_{W}^{p}\left(0, T ; L^{p}(\partial D)\right)$.

Proof. In order to prove the theorem we have to show that the following quantity is finite:

$$
\|\tau(Z)\|_{L_{W}^{p}\left(0, T ; L^{p}(\partial D)\right)}^{p}=\int_{0}^{T} \mathbb{E}\|\tau(Z)\|_{L^{p}(\partial D)}^{p} \mathrm{~d} t=\int_{0}^{T} \int_{\partial D} \mathbb{E}|Z(t, \xi)|^{p} \mathrm{~d} \xi \mathrm{d} t .
$$

So it is sufficient to prove that

$$
\mathbb{E}|Z(t, \xi)|^{p} \leq C<\infty
$$

where the constant $C$ does not depend on the variables $t$ and $\xi$. Using the same notation as in the proof of Proposition 9, let us write

$$
Z(t, \xi)=\int_{0}^{t} \int_{S} \phi(t-s, \xi, \sigma) \mathrm{d} B(\sigma, s)=\mathcal{I}(\phi)(t, \xi) .
$$

Recalling now that the Burkholder-Davis-Gundy's inequality reads, for the fractional Brownian motion case, as

$$
\mathbb{E}|\mathcal{I}(\phi)(t, \xi)|^{p} \leq\left(\int_{0}^{t} \int_{S}\left|\left(K^{*}\left(\phi(t-\cdot, \xi, \sigma) \mathbf{1}_{(0, t)}(\cdot)\right)\right)(s)\right|^{2} \mu(\mathrm{d} \sigma) \mathrm{d} s\right)^{\frac{p}{2}}
$$


and using the estimate (11), we get

$$
\begin{aligned}
\mathbb{E}|Z(t, \xi)|^{p} & =\mathbb{E}|\mathcal{I}(\phi)(t, \xi)|^{p}=\mathbb{E}\left|\int_{0}^{t} \int_{S} \phi(t-s, \xi, \sigma) \mathrm{d} B(\sigma, s)\right|^{p} \\
& \leq\left(\int_{0}^{t} \int_{S}\left|\left(K^{*}\left(\phi(t-\cdot, \xi, \sigma) \mathbf{1}_{(0, t)}(\cdot)\right)\right)(s)\right|^{2} \mu(\mathrm{d} \sigma) \mathrm{d} s\right)^{\frac{p}{2}} \\
& \leq\left(\int_{0}^{t} \int_{S}\left|\int_{s}^{t}\left(\frac{s}{r}\right)^{\frac{1}{2}-H}(r-s)^{H-\frac{3}{2}} \phi(t-r, \xi, \sigma) \mathrm{d} r\right|^{2} \mu(\mathrm{d} \sigma) \mathrm{d} s\right)^{\frac{p}{2}} \\
& =\left(\int_{0}^{t} \int_{S}\left|\int_{s}^{t}\left(\frac{s}{r}\right)^{\frac{1}{2}-H}(r-s)^{H-\frac{3}{2}}\left(\int_{\partial D} p_{N}(t-r, \xi, \bar{y}) \alpha(\sigma, \bar{y}) \mathrm{d} \bar{y}\right) \mathrm{d} r\right|^{2} \mu(\mathrm{d} \sigma) \mathrm{d} s\right)^{\frac{p}{2}} \\
& \leq\left(\int_{0}^{t} \int_{S}\left|\int_{s}^{t}\left(\frac{s}{r}\right)^{\frac{1}{2}-H}(r-s)^{H-\frac{3}{2}}\left(\int_{\partial D} c(t-r)^{-\mu}|\xi-\bar{y}|^{2 \mu-d} \alpha(\sigma, \bar{y}) \mathrm{d} \bar{y}\right)^{2} \mathrm{~d} r\right|^{2} \mu(\mathrm{d} \sigma) \mathrm{d} s\right)^{\frac{p}{2}} \\
& =c\left(\int_{0}^{t}\left(\int_{S}\left(\int_{\partial D}|\xi-\bar{y}|^{2 \mu-d} \alpha(\sigma, \bar{y}) \mathrm{d} \bar{y}\right)^{2} \mu(\mathrm{d} \sigma)\right)^{t}\left|\int_{s}^{t}\left(\frac{s}{r}\right)^{\frac{1}{2}-H}(r-s)^{H-\frac{3}{2}}(t-r)^{-\mu} \mathrm{d} r\right|^{2} \mathrm{~d} s\right)^{\frac{p}{2}} \\
& =c\left(\int_{S}\left(\int_{\partial D}|\xi-\bar{y}|^{2 \mu-d} \alpha(\sigma, \bar{y}) \mathrm{d} \bar{y}\right)^{2} \mu(\mathrm{d} \sigma)\right)^{\frac{p}{2}}\left(\int_{0}^{t}\left|\int_{s}^{t}\left(\frac{s}{r}\right)^{\frac{1}{2}-H}(r-s)^{H-\frac{3}{2}}(t-r)^{-\mu} \mathrm{d} r\right|^{2} \mathrm{~d} s\right)^{\frac{p}{2}}
\end{aligned}
$$

With the same computations used in the proof of Proposition 9, we recover the following estimate for the time variable integral

$$
\left(\int_{0}^{t}\left|\int_{s}^{t}\left(\frac{s}{r}\right)^{\frac{1}{2}-H}(r-s)^{H-\frac{3}{2}}(t-r)^{-\mu} \mathrm{d} r\right|^{2} \mathrm{~d} s\right)^{\frac{p}{2}} \leq C_{\mu, H, p} T^{p(H-\mu)}
$$

for $\mu \in\left(\frac{1}{2}, H\right)$.

As regards the space variable integral, using Lemma 27, we get

$$
\left(\int_{S}\left(\int_{\partial D}|\xi-\bar{y}|^{2 \mu-d} \alpha(\sigma, \bar{y}) \mathrm{d} \bar{y}\right)^{2} \mu(\mathrm{d} \sigma)\right)^{\frac{p}{2}} \leq C\|\alpha\|_{L^{\theta}\left(\partial D ; L^{2}(S)\right)}^{p}
$$

Hence we have

$$
\mathbb{E}|Z(t, \xi)|^{p} \leq C_{\mu, p, H} T^{p(H-\mu)}\|\alpha\|_{L^{\theta}\left(\partial D ; L^{2}(S)\right)}^{p} .
$$

Since the constant does not depend on $t$ and $\xi$ we get the thesis.

Remark 3. Let us notice that we have the continuity in space of the convolution process only inside the domain D. For what concerns the spatial regularity of the process on the boundary we can only give a meaning of its trace as an element of $L^{p}(\partial D)$. So, on the boundary, we do not have enough regularity in order to evaluate the process pointwise in space. Anyway, as pointed out in the Introduction, in the following we will consider $Z(t, \xi)$ for $\xi \in \partial D$ (and then $u(t, \xi)$ ), remembering that these are only representative elements in the class $\left.L^{p}(\partial D)\right)$, and then all the stated results are valid only a.e. in space. 
4.2. Malliavin derivative of the convolution process and existence of a density. We next analyze the Malliavin derivative of the process $Z(t, x)$

$$
D_{s, \sigma} Z(t, x)=\int_{\partial D} p_{N}(t-r, x, y) \alpha(\sigma, \bar{y}) \mathbb{1}_{[0, t]}(r) \mathrm{d} \bar{y} .
$$

As explained in Remark 3, we can study pointwise in space the Malliavin derivative of the random variable $Z(t, x)$ for every $x$ inside the domain. But, as a necessary step, we need to study the Malliavin derivative computed for points on the boundary, and this study has to be interpreted in an a.e. sense.

Lemma 12. Under assumption (a1'), the random variable $Z(t, x)$ belongs to $\mathbb{D}^{1,2}$ for any $(t, x) \in$ $(0, T) \times \bar{D}$.

Proof. In view of Lemma 11, Proposition 9, and Remark 2, it remains to prove that the Malliavin derivative belongs to $\mathcal{H}$.

The key point in the following computation is provided by the estimate (11)

$$
p_{N}(t, x, \bar{y}) \leq C t^{-\mu}|x-\bar{y}|^{-d+2 \mu}
$$

We get

$$
\begin{aligned}
\|D Z(t, x)\|_{\mathcal{H}}^{2} & =\int_{0}^{t} \int_{0}^{t} \int_{S} D_{s, \sigma} Z(t, x) D_{r, \sigma} Z(t, x)|s-r|^{2 H-2} \mu(\mathrm{d} \sigma) \mathrm{d} r \mathrm{~d} s \\
& =\int_{0}^{t} \int_{0}^{t} \int_{S}\left(\int_{\partial D} p_{N}(t-s, x, \bar{y}) \alpha(\sigma, \bar{y}) \mathrm{d} \bar{y}\right)\left(\int_{\partial D} p_{N}(t-r, x, \bar{y}) \alpha(\sigma, \bar{y}) \mathrm{d} \bar{y}\right)|s-r|^{2 H-2} \mu(\mathrm{d} \sigma) \mathrm{d} r \mathrm{~d} s \\
& \leq C \int_{0}^{t} \int_{0}^{t} \int_{S}\left(\int_{\partial D}|x-\bar{y}|^{-d+2 \mu}|\alpha(\sigma, \bar{y})| \mathrm{d} \bar{y}\right)^{2}(t-s)^{-\mu}(t-r)^{-\mu}|s-r|^{2 H-2} \mu(\mathrm{d} \sigma) \mathrm{d} r \mathrm{~d} s
\end{aligned}
$$

hence we can separately examine the spatial and the time integrals. First, we have

$$
\begin{aligned}
\int_{0}^{t} \int_{0}^{t}(t-s)^{-\mu}(t-r)^{-\mu}|s-r|^{2 H-2} \mathrm{~d} r \mathrm{~d} s & =2 \int_{0}^{t} s^{-\mu}\left(\int_{0}^{s} r^{-\mu}(s-r)^{2 H-2} \mathrm{~d} r\right) \mathrm{d} s \\
& =2 \int_{0}^{t} s^{-\mu}\left(C_{H, \mu} s^{2 H-1-\mu}\right) \mathrm{d} s=C_{H, \mu}^{\prime} t^{2 H-2 \mu}
\end{aligned}
$$

In order to handle the spatial term, we have to consider separately the cases $x \in \stackrel{D}{D}$ and $x \in \partial D$. Let us at first consider the case $x \in \stackrel{\circ}{D}$. In this case $\bar{y} \mapsto|x-\bar{y}|^{-d+2 \mu}$ belongs to $L^{2}(\partial D)$. In fact, denoting $\varepsilon=\operatorname{dist}(x, \partial D)$ and $\Gamma=\operatorname{diam}(D)$ the diameter of $D$, then

$$
\varepsilon \leq|x-\bar{y}| \leq \Gamma
$$

and so

$$
\int_{\partial D}|x-\bar{y}|^{2(-d+2 \mu)} \mathrm{d} \bar{y} \leq \int_{\partial D} \max \left\{\varepsilon^{-2 d+4 \mu}, \Gamma^{-2 d+4 \mu}\right\} \mathrm{d} \bar{y}<+\infty .
$$

Therefore, we get

$$
\int_{S}\left(\int_{\partial D}|x-\bar{y}|^{-d+2 \mu}|\alpha(\sigma, \bar{y})| \mathrm{d} \bar{y}\right)^{2} \mu(\mathrm{d} \sigma) \leq C\|\alpha\|_{L^{2}(\partial D \times S)}^{2}
$$


If $x \in \partial D$, thanks to Lemma 27 we have that

$$
\int_{S}\left(\int_{\partial D}|x-\bar{y}|^{-d+2 \mu}|\alpha(\sigma, \bar{y})| \mathrm{d} \bar{y}\right)^{2} \mu(\mathrm{d} \sigma) \leq C\|\alpha\|_{L^{\theta}\left(\partial D ; L^{2}(S)\right.}^{2}
$$

which allows to conclude

$$
\|D Z(t, x)\|_{\mathcal{H}}^{2}<+\infty
$$

as required.

The following result is a refinement of the previous Lemma.

Lemma 13. The random variable $Z(t, x)$ belongs to $\mathbb{D}^{1, p}$ for any $(t, x) \in(0, T) \times \bar{D}$ and for every $p>2$.

Proof. We have only to prove that

$$
\mathbb{E}\|D Z(t, x)\|_{\mathcal{H}}^{p}<\infty
$$

This easily follows from Lemma 12, In fact

$$
\mathbb{E}\|D Z(t, x)\|_{\mathcal{H}}^{p}=\|D Z(t, x)\|_{\mathcal{H}}^{p}=\left(\|D Z(t, x)\|_{\mathcal{H}}^{2}\right)^{\frac{p}{2}}
$$

and so, if $x \in D$ we recover

$$
\mathbb{E}\|D Z(t, x)\|_{\mathcal{H}}^{p} \leq\left(C_{H, \mu}^{\prime} T^{2 H-2 \mu} C\|\alpha\|_{L^{2}(\partial D \times S)}^{2}\right)^{\frac{p}{2}}<\infty,
$$

whereas for the case $\xi \in \partial D$ we get

$$
\mathbb{E}\|D Z(t, \xi)\|_{\mathcal{H}}^{p} \leq\left(C_{H, \mu}^{\prime} T^{2 H-2 \mu} C_{|\partial D|}\right)^{\frac{p}{2}}<\infty .
$$

Lemma 14. The stochastic convolution process belongs to $\mathbb{D}^{\infty}$.

Proof. Simply notice that $D \delta(u)=u$ for any deterministic function $u \in \mathcal{H}$, where we use the notation $\delta(u)$ for the Wiener integral. Therefore, higher order derivatives vanishes and the thesis follows.

We finally prove the existence of the density of the random variable $Z(t, x)$ with respect to the Lebesgue measure on $\mathbb{R}$. We shall use the criterion for absolute continuity stated in Theorem 7 .

Lemma 15. Under the non-degeneracy hypothesis (a2), the random variable $Z(t, x),(t, x) \in[0, T] \times D$ has a smooth density with respect to the Lebesgue measure on $\mathbb{R}$.

Proof. The thesis follows once we provide a constant $\delta$ that satisfies the estimate

$$
\|D Z(t, x)\|_{\mathcal{H}}^{2} \geq \delta>0 \quad \text { a.s. }
$$


So we compute

$$
\begin{aligned}
\|D Z(t, x)\|_{\mathcal{H}}^{2}= & \int_{0}^{t} \int_{0}^{t} \int_{S} D_{s, \sigma} Z(t, x) D_{r, \sigma} Z(t, x)|s-r|^{2 H-2} \mu(\mathrm{d} \sigma) \mathrm{d} r \mathrm{~d} s \\
= & \int_{0}^{t} \int_{0}^{t} \int_{S}\left(\int_{\partial D} p_{N}(t-s, x, \bar{y}) \alpha(\sigma, \bar{y}) \mathrm{d} \bar{y}\right)\left(\int_{\partial D} p_{N}(t-r, x, \bar{y}) \alpha(\sigma, \bar{y}) \mathrm{d} \bar{y}\right)|s-r|^{2 H-2} \mu(\mathrm{d} \sigma) \mathrm{d} r \mathrm{~d} s \\
& \geq C_{1}^{2} \int_{0}^{t} \int_{0}^{t}(t-s)^{-\frac{d}{2}}(t-r)^{-\frac{d}{2}}|s-r|^{2 H-2} \times \\
& \times\left[\int_{S}\left(\int_{\partial D} e^{-c_{2} \frac{|x-\bar{y}|^{2}}{t-s}} \alpha(\sigma, \bar{y}) \mathrm{d} \bar{y}\right)\left(\int_{\partial D} e^{-c_{2} \frac{|x-\bar{y}|^{2}}{t-r}} \alpha(\sigma, \bar{y}) \mathrm{d} \bar{y}\right) \mu(\mathrm{d} \sigma)\right] \mathrm{d} r \mathrm{~d} s
\end{aligned}
$$

where we have used the lower bound estimate for the Poisson kernel (see Lemma 4). Let us now fix $\varepsilon>0$ and let $s<t-\varepsilon^{2}$. We then have the following estimate

$$
\begin{aligned}
\int_{\partial D} e^{-c_{2} \frac{|x-\bar{y}|^{2}}{t-s}} \alpha(s, \bar{y}) \mathrm{d} \bar{y} & \geq \int_{\partial D \cap B(x, \varepsilon)} e^{-c_{2} \frac{|x-\bar{y}|^{2}}{t-s}} \alpha(s, \bar{y}) \mathrm{d} \bar{y} \\
& \geq c \alpha_{0} \varepsilon^{d-1} e^{-\frac{\varepsilon^{2}}{t-s}} \geq c \alpha_{0} \varepsilon^{d-1} e^{-c_{2}}=C_{\alpha_{0}, c_{2}} \varepsilon^{d-1}
\end{aligned}
$$

Choosing $\varepsilon^{2}=\frac{t}{2}$ we then have

$$
\begin{aligned}
\|D Z(t, x)\|_{\mathcal{H}}^{2} \geq & C_{\alpha_{0}, c_{2}, c_{1},|S|} t^{d-1} \int_{0}^{\frac{t}{2}} \int_{0}^{\frac{t}{2}}(t-s)^{-\frac{d}{2}}(t-r)^{-\frac{d}{2}}|s-r|^{2 H-2} \mathrm{~d} r \mathrm{~d} s \\
& =C_{\alpha_{0}, c_{2}, c_{1},|S|} t^{d-1} \int_{0}^{\frac{t}{2}} \int_{0}^{\frac{t}{2}}\left(\sigma+\frac{t}{2}\right)^{-\frac{d}{2}}\left(\rho+\frac{t}{2}\right)^{-\frac{d}{2}}|\sigma-\rho|^{2 H-2} \mathrm{~d} \sigma \mathrm{d} \rho \\
& \geq C_{\alpha_{0}, c_{2}, c_{1},|S|} t^{-1} \int_{0}^{\frac{t}{2}} \int_{0}^{\frac{t}{2}}|\sigma-\rho|^{2 H-2} \mathrm{~d} \sigma \mathrm{d} \rho \geq \tilde{C} t^{2 H-1}>0
\end{aligned}
$$

as required.

\section{The SOlution of THE NONLINEAR PROBlem}

We consider in this section the non homogeneous diffusion equation (1). In the first part of the section we impose condition (g1) on the function $g$ :

$g$ is Lipschitz continuous and belongs to the class $C^{1}(\mathbb{R})$ with $\left|\partial_{u} g(u)\right| \leq L$,

$$
g(u) \leq c(1+|u|) \text { for some finite constant } L>0 .
$$

5.1. Existence and global regularity of the solution. While dealing with the problem of the existence and uniqueness of the solution, we shall apply the same ideas of [1, Section 3.2]. We first consider the Volterra equation on $[0, T] \times \partial D$,

$$
u(t, \xi)=\int_{0}^{t} \int_{\partial D} p_{N}(t-s, \xi, \bar{y}) g(u(s, \bar{y})) \mathrm{d} \bar{y} \mathrm{~d} s+\int_{0}^{t} \int_{S} \int_{\partial D} p_{N}(t-s, \xi, \bar{y}) \alpha(\sigma, \bar{y}) \mathrm{d} \bar{y} \mathrm{~d} B(\sigma, s) .
$$

Theorem 16. Assume that Hypothesis (g1) holds. Then equation (21) has a unique solution in the space $L_{W}^{p}\left([0, T] ; L^{p}(\partial D)\right)$. 
Proof. For the proof we follow the ideas of [1, Lemma 3.4]. The existence of a solution follows from a fixed point argument. Let us introduce in the space $L_{W}^{p}\left([0, T] ; L^{p}(\partial D)\right)$ the equivalent norm

$$
\|f\|_{L_{W}^{p}}=\mathbb{E} \int_{0}^{T} e^{-\lambda t}\|f(t)\|_{L^{p}(\partial D)}^{p} \mathrm{~d} t
$$

then we introduce the operator

$$
\Phi(u)(t, \xi)=\int_{0}^{t} \int_{\partial D} p_{N}(t-s, \xi, \bar{y}) g(u(s, \bar{y})) \mathrm{d} \bar{y} \mathrm{~d} s+Z(t, \xi)
$$

and prove that it is a well-defined mapping from the space $L_{W}^{p}\left([0, T] ; L^{p}(\partial D)\right)$ into itself. Moreover, it is a contraction for some suitable $\lambda$. Thanks to Lemma 11, this will follow from the following estimate

$$
\begin{aligned}
\|\Phi(u)-\Phi(v)\|_{L_{W}^{p}}^{p} & =\mathbb{E} \int_{0}^{T} e^{-\lambda t} \int_{\partial D}\left|\int_{0}^{t} \int_{\partial D} p_{N}(t-s, \xi, \bar{y})(g(u(s, \bar{y}))-g(v(s, \bar{y}))) \mathrm{d} \bar{y} \mathrm{~d} s\right|^{p} \mathrm{~d} \xi \mathrm{d} t \\
& \leq L^{p} \mathbb{E} \int_{0}^{T} e^{-\lambda t} \int_{\partial D}\left(\int_{0}^{t} \int_{\partial D} p_{N}(t-s, \xi, \bar{y})|u(s, \bar{y})-v(s, \bar{y})| \mathrm{d} \bar{y} \mathrm{~d} s\right)^{p} \mathrm{~d} \xi \mathrm{d} t
\end{aligned}
$$

Now, proceeding exactly as in the proof of Lemma 30, we recover

$$
\|\Phi(u)-\Phi(v)\|_{L_{W}^{p}}^{p} \leq C \lambda^{\frac{\mu-1}{p-1}}\|u-v\|_{L_{W}^{p}}^{p}
$$

Then we see that there exists $\lambda$ large enough such that $C \lambda^{\frac{\mu-1}{p-1}}<1-\varepsilon<1$ and this proves the Theorem.

Given the process $\varphi \in L_{W}^{p}\left([0, T] ; L^{p}(\partial D)\right)$ that is the solution of problem (21), the solution of the original problem (3) is given by the representation formula

$$
u(t, x)=\int_{0}^{t} \int_{\partial D} p_{N}(t-s, x, \bar{y}) g(\varphi(s, \bar{y})) \mathrm{d} \bar{y} \mathrm{~d} s+\int_{0}^{t} \int_{S} \int_{\partial D} p_{N}(t-s, x, \bar{y}) \alpha(\sigma, \bar{y}) \mathrm{d} \bar{y} \mathrm{~d} B(\sigma, s) .
$$

Corollary 17. For every $t \in[0, T]$ and almost surely, the solution $u(t, x)$ of problem (3) is a continuous function for $x \in D$.

Corollary 18. There exists a continuous modification $u=\{u(t, x), t \in[0, T] \times D\}$ of the solution process (22).

5.2. The Malliavin derivative of the solution. We are concerned with the law of the random variable $u(t, x)$ that represents the solution of problem (3) for $t \in(0, T)$ and $x \in D$. We shall prove first that $u(t, x)$ has a density that is absolutely continuous with respect to the Lebesgue measure. Later in this section we shall prove, under additional conditions on the coefficients of (3), that this density is smooth $\left(C^{\infty}\right)$.

Next result concerns the existence of the Malliavin derivative for the solution $u$. Heuristically, the Malliavin derivative is the solution of the problem that we get by formally taking derivative in the original problem (4); however, to make the construction complete, we shall appeal to the Picard's approximations of $u$ and apply Proposition 8 . 
Theorem 19. Assume that Hypothesis (g1) and (a1') hold. Then the Malliavin derivative of the solution process $D u$ belongs to the space $L_{W}^{p}((0, T) \times \partial D ; \mathcal{H})$ and it satisfies the following equation:

$$
\begin{aligned}
D_{r, \sigma} u(t, \xi)=\int_{\partial D} p_{N}(t-r, \xi, \bar{y}) \alpha(\sigma, \bar{y}) \mathbf{1}_{[0, t]}(r) \mathrm{d} \bar{y} & \\
& +\int_{r}^{t} \int_{\partial D} p_{N}(t-s, \xi, \bar{y}) \partial_{u} g(u(s, \bar{y})) D_{r, \sigma} u(s, \bar{y}) \mathrm{d} \bar{y} \mathrm{~d} s .
\end{aligned}
$$

Proof. Let us consider the sequence of Picard's approximations

$$
\begin{aligned}
u_{0}(t, \xi) & =\int_{0}^{t} \int_{S} \int_{\partial D} p_{N}(t-s, \xi, \bar{y}) \alpha(\sigma, \bar{y}) \mathrm{d} \bar{y} \mathrm{~d} B(\sigma, s), \\
u_{n+1}(t, \xi) & =u_{0}(t, \xi)+\int_{0}^{t} \int_{\partial D} p_{N}(t-s, \xi, \bar{y}) g\left(u_{n}(s, \bar{y})\right) \mathrm{d} \bar{y} \mathrm{~d} s .
\end{aligned}
$$

From Lemma 11 we know that $u_{0} \in L_{W}^{p}\left(0, T ; L^{p}(\partial D)\right)$. Using then the Hypothesis (g1) and appealing to Lemma 30 (in the case $k=0$ ), immediately follows that

$$
\left\|u_{n+1}-u_{n}\right\| \leq C\left\|u_{n}-u_{n-1}\right\|
$$

for a constant $C<1$. So we obtain that the sequence $u_{n}$ converge to $u$, which is clearly the solution of (4), in $L_{W}^{p}\left(0, T ; L^{p}(\partial D)\right)$ and $\sup _{n}\left\|u_{n}\right\|<\infty$.

It remains to prove that

$$
\sup _{n}\left\|D u_{n}(t, \xi)\right\|<\infty
$$

which allows to apply Proposition 8 and get the thesis.

For any $n \in \mathbb{N}$, taking the Malliavin derivative in the equation defining $u_{n+1}$ we get

$$
\begin{aligned}
D_{r, \sigma} u_{n+1}(t, \xi)=\int_{0}^{t} \int_{\partial D} p_{N}(t-s, \xi, \bar{y}) \partial_{u} g\left(u_{n}(s, \bar{y})\right) D_{r, \sigma} u_{n}(s, \bar{y}) \mathrm{d} \bar{y} \mathrm{~d} s & \\
& \quad+\int_{\partial D} p_{N}(t-r, \xi, \bar{y}) \alpha(\sigma, \bar{y}) \mathbf{1}_{[0, t]}(r) \mathrm{d} \bar{y} .
\end{aligned}
$$

Let us introduce on the space $L_{W}^{p}((0, T) \times \partial D ; \mathcal{H})$ the equivalent norm

$$
\|f\|_{L_{W}^{p}}^{p}=\mathbb{E} \int_{0}^{T} e^{-\lambda t}\|f(t)\|_{L^{p}(\partial D ; \mathcal{H})}^{p} \mathrm{~d} t
$$

for some constant $\lambda$ to be chosen later. This norm is equivalent to the standard one. Let us define

$$
\begin{aligned}
\varphi_{0}(r, \sigma, t, \xi) & :=\int_{\partial D} p_{N}(t-r, \xi, \bar{y}) \alpha(\sigma, \bar{y}) \mathbf{1}_{[0, t]}(r) \mathrm{d} \bar{y} \\
\varphi_{n+1}(r, \sigma, t, \xi) & :=\varphi_{0}(r, \sigma, t, \xi)+\int_{r}^{t} \int_{\partial D} p_{N}(t-s, \xi, \bar{y}) \partial_{u} g\left(u_{n}(s, \bar{y})\right) \varphi_{n}(r, \sigma, s, \bar{y}) \mathrm{d} \bar{y} \mathrm{~d} s .
\end{aligned}
$$

We aim to prove that, for every $n, \varphi_{n} \in L_{W}^{p}([0, T] \times \partial D ; \mathcal{H})$ and

$$
\left\|\varphi_{n+1}-\varphi_{n}\right\|_{L_{W}^{p}([0, T] \times \partial D ; \mathcal{H})} \leq C\left\|\varphi_{n}-\varphi_{n-1}\right\|_{L_{W}^{p}([0, T] \times \partial D ; \mathcal{H})}
$$


for a suitable constant $C<1$. Since this result is mainly a technical tool and requires some computation, we pospone its proof to Lemma 30 in the Appendix. Now, assume that (26) is given. Then

$$
\begin{aligned}
\left\|\varphi_{n+1}\right\| & \leq\left\|\varphi_{n+1}-\varphi_{0}\right\|+\left\|\varphi_{0}\right\|=\sum_{k=0}^{n}\left\|\varphi_{k+1}-\varphi_{k}\right\|+\left\|\varphi_{0}\right\| \\
& \leq \sum_{k=0}^{n} C^{k}\left\|\varphi_{0}\right\|+\left\|\varphi_{0}\right\| \leq\left(\frac{1}{1-C}+1\right)\left\|\varphi_{0}\right\|<\infty
\end{aligned}
$$

holds for any $n$. This allows to apply Proposition 8 and prove that $u \in \mathbb{D}^{1, p}$. Moreover, since $D u_{n}$ converges weakly to $D u$, then we pass to the limit in the expression of the Malliavin derivative of $u_{n}$ and we obtain equation (23).

The Malliavin derivative of the solution $u(t, x)$ for problem (3),$x \in D$, is obtained from the process on the boundary, as explained in the following result.

Proposition 20. Given the random variable $\varphi \in L_{W}^{2}([0, T] \times \partial D ; \mathcal{H})$ that is the solution of (23), the solution of

$D_{r, z} u(t, x)=\int_{\partial D} p_{N}(t-r, x, \bar{y}) \alpha(\sigma, \bar{y}) \mathbf{1}_{[0, t]}(r) \mathrm{d} \bar{y}+\int_{r}^{t} \int_{\partial D} p_{N}(t-s, x, \bar{y}) \partial_{u} g(u(s, \bar{y})) D_{r, z} u(s, \bar{y}) \mathrm{d} \bar{y} \mathrm{~d} s$ on $[0, T] \times D$ is given by the representation formula

$$
D_{r, z} u(t, x)=\int_{\partial D} p_{N}(t-r, x, \bar{y}) \alpha(\bar{y}) \mathbf{1}_{[0, t]}(r) \mathrm{d} \bar{y}+\int_{r}^{t} \int_{\partial D} p_{N}(t-s, x, \bar{y}) \partial_{u} g(u(s, \bar{y})) \varphi(r, \sigma, s, \bar{y}) \mathrm{d} \bar{y} \mathrm{~d} s
$$

5.3. Smoothness of the Malliavin derivative. In the sequel we prove that, under the stronger assumption (g2), the solution of problem (4) belongs to $\mathbb{D}^{\infty}$. In turn, this result will allow to study the smoothness of the density of the random variable $u(t, x)$ with respect to the Lebesgue measure.

Let us give an hint about the following construction. First, notice that $u \in \mathbb{D}^{\infty}$ means that $u \in \mathbb{D}^{k, p}$ for any $k \in \mathbb{N}$ and $p \geq 1$; moreover, it is also implied if we prove that $D^{k} u \in \mathbb{D}^{1, p}$ for any $k$ and any $p$. Taking a look to the equation satisfied by the Malliavin derivative $D u(t, x)$, we notice that it has the same form as the original problem (4), just changing the relevant coefficients. Then we may use this analogy to prove that this process has a derivative itself. After that, we shall use an iteration argument to conclude the construction.

For the sake of simplicity, we shall provide a unique result concerning existence of the Malliavin derivative of the solution for a general class of problems.

Lemma 21. Let $V(t, x)$ the solution of the linear (integral) equation in $\mathcal{H}^{\otimes k}$, for every $k \geq 1$,

$$
V(t, x)=f_{k}(t, x)+\int_{0}^{t} \int_{\partial D} p_{N}(t-s, x, \bar{y}) h(s, \bar{y}) V(s, \bar{y}) \mathrm{d} s \mathrm{~d} \bar{y} .
$$

Under the assumptions (for every $p \geq 2$ )

$$
\begin{gathered}
f_{k} \in L_{W}^{p}\left((0, T) \times \partial D ; \mathcal{H}^{\otimes k}\right), \\
h \in L_{W}^{\infty}((0, T) \times \partial D), \\
D f_{k} \in L_{W}^{p}\left((0, T) \times \partial D ; \mathcal{H}^{\otimes(k+1)}\right), \\
D h \in L_{W}^{\infty}((0, T) \times \partial D ; \mathcal{H}),
\end{gathered}
$$


we have that $V(t, x) \in \mathbb{D}^{1, \infty}$ for a.e. $(t, x)$ and

$$
D V(t, x)=f_{k+1}(t, x)+\int_{0}^{T} \int_{\partial D} p_{N}(t-s, x, \bar{y}) h(s, \bar{y}) D V(s, \bar{y}) \mathrm{d} s \mathrm{~d} \bar{y}
$$

where

$$
f_{k+1}(t, x)=D f_{k}(t, x)+\int_{0}^{T} \int_{\partial D} p_{N}(t-s, x, \bar{y}) D h(s, \bar{y}) V(s, \bar{y}) \mathrm{d} \bar{y} \mathrm{~d} s
$$

Proof. For the sake of simplicity let us divide the proof in four steps.

Step 1: Consider the Picard approximations defined by the recursive equations:

$$
\begin{gathered}
V_{0}(t, x)=f_{k}(t, x) \\
V_{n+1}(t, x)=V_{0}(t, x)+\int_{0}^{T} \int_{\partial D} p_{N}(t-s, x, \bar{y}) h(s, \bar{y}) V_{n}(s, \bar{y}) \mathrm{d} s \mathrm{~d} \bar{y}
\end{gathered}
$$

Notice that the term $V_{0} \in L_{W}^{p}\left((0, T) \times \partial D ; \mathcal{H}^{\otimes k}\right)$ by assumption. Since $h$ is bounded and $p_{N}$ is a Gaussian kernel, it is possible to prove (use Lemma 30) that the right hand side of equation (35) defines a contraction in the space $L_{W}^{p}\left((0, T) \times \partial D ; \mathcal{H}^{\otimes k}\right)$, for every $p \geq 2$. This implies the existence and uniqueness of the random variable $V \in \bigcap_{p \geq 2} L_{W}^{p}\left((0, T) \times \partial D ; \mathcal{H}^{\otimes k}\right)$ which is the solution of (28).

Step 2: Taking the Malliavin derivative in (35) we get

$$
\begin{aligned}
D V_{n+1}(t, x)= & D f_{k}(t, x)+\int_{0}^{t} \int_{\partial D} p_{N}(t-s, x, \bar{y}) D h(s, \bar{y}) V_{n}(s, \bar{y}) \mathrm{d} \bar{y} \mathrm{~d} s \\
& +\int_{0}^{t} \int_{\partial D} p_{N}(t-s, x, \bar{y}) h(s, \bar{y}) D V_{n}(s, \bar{y}) \mathrm{d} \bar{y} \mathrm{~d} s \\
= & \lambda_{n}(t, x)+\int_{0}^{t} \int_{\partial D} p_{N}(t-s, x, \bar{y}) h(s, \bar{y}) D V_{n}(s, \bar{y}) \mathrm{d} \bar{y} \mathrm{~d} s .
\end{aligned}
$$

We see that, for every $n, \lambda_{n} \in L_{W}^{p}\left((r, T) \times \partial D ; \mathcal{H}^{\otimes(k+1)}\right)$. This follows from assumptions (29) - (31) and by the fact (proved in Step 1 ) that $V_{n} \in L_{W}^{p}\left((r, T) \times \partial D ; \mathcal{H}^{\otimes k}\right)$ for every $n$. So, proceeding exactly as in step 1 we get that the right hand side of the above expression defines a contraction in the space $L_{W}^{p}\left((0, T) \times \partial D ; \mathcal{H}^{\otimes(k+1)}\right)$.

Step 3: At this point we recover the following estimate:

$$
\sup _{n}\left\|D V_{n}\right\|_{L_{W}^{p}\left((0, T) \times \partial D ; \mathcal{H}^{\otimes(k+1)}\right)} \leq C<\infty,
$$

In fact, we have:

$$
\left\|D V_{n}\right\| \leq\left\|D V_{0}\right\|+\left\|D V_{n}-D V_{0}\right\| \leq\left\|D V_{0}\right\|+\sum_{j=0}^{n-1}\left\|D V_{j+1}-D V_{j}\right\|,
$$

where $\left\|D V_{0}\right\|<C$ since $D V_{0} \in L_{W}^{p}\left((0, T) \times \partial D ; \mathcal{H}^{\otimes(k+1)}\right)$ by step 3 and, thanks to Lemma 30 we recover

$$
\begin{aligned}
\left\|D V_{j+1}-D V_{j}\right\| & \leq\left\|\lambda_{j}-\lambda_{j-1}\right\|+\left\|\int_{r}^{t} \int_{\partial D} p_{N}(t-s, x, \bar{y}) h(s, \bar{y})\left(D V_{j}-D V_{j-1}\right)(s, \bar{y}) \mathrm{d} \bar{y} \mathrm{~d} s\right\| \\
& \leq C\left\|V_{j}-V_{j-1}\right\|+C\left\|D V_{j}-D V_{j-1}\right\|
\end{aligned}
$$


Iterating this inequality we recover

$$
\begin{aligned}
\sum_{j=0}^{n-1}\left\|D V_{j+1}-D V_{j}\right\| & \leq\left\|V_{1}-V_{0}\right\| \sum_{j=0}^{n-1} j C^{j}+\left\|D V_{1}-D V_{0}\right\| \sum_{j=0}^{n-1} C^{j} \\
& \leq \frac{1}{(1-C)^{2}}\left\|V_{1}-V_{0}\right\|+\frac{1}{1-C}\left\|D V_{1}-D V_{0}\right\|
\end{aligned}
$$

which allows us to conclude that estimate (37) holds.

Step 4: Appealing to Proposition 8 , by steps one and three it follows that $V \in \mathbb{D}^{1, p}$ for every $p \geq 2$. Since $V_{n} \rightarrow V$ and $D V_{n} \rightarrow D V$, passing to the limit in (36) we get that $D V$ solves (33).

Remark 4. In our case, we recognize that

$$
h(t, x)=\partial_{u} g(u(t, x))
$$

is a bounded process and, by considering (23), we may set

$$
f_{1}(t, x)=\int_{\partial D} p_{N}(t-r, \xi, \bar{y}) \alpha(\sigma, \bar{y}) \mathbf{1}_{[0, t]}(r) \mathrm{d} \bar{y}
$$

which satisfies the assumption of Lemma 21. We may also compute

$$
f_{2}(t, x)=\iint p_{N}(t-s, x, y) \partial_{u}^{2} g(u(s, y))(D u(s, y))^{2} \mathrm{~d} y \mathrm{~d} s
$$

and, in general, $f_{n+1}$ depends on $\partial_{u}^{2} g, \ldots, \partial_{u}^{n+1} g$, and $D u, \ldots, D^{n} u$ in a polynomial way, so a recursive argument implies that the assumption of Lemma 21] are satisfied for any $n$.

Corollary 22. The random variable $u(t, x)$ belongs to the space $\mathbb{D}^{\infty}$ for every $(t, x) \in[0, T] \times \bar{D}$.

Proof. First, as explained above, the result follows for $x \in \partial D$ from Lemma 21 and Remark 4 . Then, we conclude by noticing that for $x \in D$ the Malliavin derivative is given (compare with Proposition 20) in terms of the same process on the boundary, hence we infer the regularity of $u(t, x)$ by that of $u(t, \xi)$ for $\xi \in \partial D$.

\section{EXISTENCE OF THE DENSITY FOR THE SOLUtion OF THE NON HOMOGENEOUS EQUATION}

We are concerned with the law of the random variable $u(t, x)$ that represents the solution of problem (3) for $t \in(0, T)$ and $x \in D$. We shall prove first that $u(t, x)$ has a density that is absolutely continuous with respect to the Lebesgue measure. Later in this section we shall prove, under additional conditions on the coefficients of (3), that this density is smooth $\left(C^{\infty}\right)$.

Theorem 23. For every $t \in[0, T]$ and a.e. $\xi \in \partial D$, the random variable $u(t, \xi)$ has a density with respect to the Lebesgue measure on $\mathbb{R}$.

Proof. In order to get some relevant estimates, we will consider a smaller time interval than $(0, t)$ and consider the $\mathcal{H}$ norm of $u(t, \xi)$ on $(t-\delta, t)$ for some $\delta>0$ small enough. Then we define, for every $\varphi \in \mathcal{H}$ the norm

It is then straightforward to get

$$
\|\varphi\|_{\mathcal{H}_{\delta}}:=\left\|\mathbf{1}_{(t-\delta, t)}(\cdot) \varphi\right\|_{\mathcal{H}}
$$

$$
\|\varphi\|_{\mathcal{H}} \geq\|\varphi\|_{\mathcal{H}_{\delta}}
$$


The existence of the density follows from Theorem 7 and the estimate

$$
\mathbb{E}\|D u(t, \xi)\|_{\mathcal{H}}^{2}>0 \quad \text { a.s. }
$$

In turn, to prove that (39) holds $\mathbb{P}$-a.s., the idea is to prove that

$$
\mathbb{P}\left(\|D u(t, \xi)\|_{\mathcal{H}}^{2}<\varepsilon\right) \rightarrow 0
$$

as $\varepsilon \rightarrow 0$.

Setting for simplicity

$$
G(t, \xi):=\int_{0}^{t} \int_{\partial D} p_{N}(t-s, \xi, \bar{y}) g(u(s, \bar{y})) \mathrm{d} \bar{y} \mathrm{~d} s
$$

we get

$$
\begin{aligned}
\|D u(t, \xi)\|_{\mathcal{H}}^{2} \geq\|D u(t, \xi)\|_{\mathcal{H}_{\delta}}^{2} & =\|D Z(t, \xi)+D G(t, \xi)\|_{\mathcal{H}_{\delta}}^{2} \\
& \geq \frac{1}{2}\|D Z(t, \xi)\|_{\mathcal{H}_{\delta}}^{2}-\|D G(t, \xi)\|_{\mathcal{H}_{\delta}}^{2} .
\end{aligned}
$$

Using then Chebyshev's inequality we have

$$
\begin{aligned}
\mathbb{P}\left(\|D u(t, \xi)\|_{\mathcal{H}}^{2}<\varepsilon\right) & \leq \mathbb{P}\left(\|D u(t, \xi)\|_{\mathcal{H}_{\delta}}^{2}<\varepsilon\right) \\
& \leq \mathbb{P}\left(\|D G(t, \xi)\|_{\mathcal{H}_{\delta}}^{2} \geq \frac{1}{2}\|D Z(t, \xi)\|_{\mathcal{H}_{\delta}}^{2}-\varepsilon\right) \\
& \leq \frac{\mathbb{E}\|D G(t, \xi)\|_{\mathcal{H}_{\delta}}^{2 \tilde{p}}}{\left(\frac{1}{2}\|D Z(t, \xi)\|_{\mathcal{H}_{\delta}}^{2}-\varepsilon\right)^{\tilde{p}}}
\end{aligned}
$$

Using the estimates obtained in Lemmas 28 and 29, and choosing $\delta$ such that

$$
\frac{1}{2} C_{T, x, \delta_{0}, \alpha} \delta^{2 H-1}=2 \varepsilon,
$$

for every $\varepsilon<\varepsilon_{0}=\frac{1}{4} C_{T, x, \delta_{0}, \alpha} \delta_{0}^{2 H-1}$ we have

$$
\mathbb{P}\left(\|D u(t, \xi)\|_{\mathcal{H}}^{2}<\varepsilon\right) \leq \frac{c \delta^{2 \tilde{p}}}{\varepsilon^{\tilde{p}}}=C\left(\varepsilon^{\frac{3-2 H}{2 H-1}}\right)^{\tilde{p}}
$$

and since $\frac{3-2 H}{2 H-1}>0$ for any $H \in\left(\frac{1}{2}, 1\right)$, the above estimate allows us to conclude the proof.

Remark 5. Thanks to Definition 20 we can extend the result proved in Theorem 23 to every $(t, x) \in$ $[0, T] \times D$. This allows us to conclude that the image law of the solution of problem (4) is absolutey continuous with respect to the Lebesgue measure on $\mathbb{R}$.

6.1. Smoothness of the density for the solution of the non homogeneous equation. In case the coefficients of the equation are more regular than just Lipschitz continuous, we can prove the smoothness of the density of the random variable $u(t, x)$ that solves Eq.(3) for $(t, x) \in[0, T] \times D$, analogously with the result in Lemma 15.

Theorem 24. Assume that the condition (g2) holds. Then for each $(t, x) \in[0, T] \times D$, the random variable $u(t, x)$ has a density with respect to the Lebesgue measure that is infinitely differentiable. 
Proof. The idea is to apply Theorem [7. Let us at first consider $u(t, \xi)$ for $(t, \xi) \in[0, T] \times \partial D$. From Corollary 22 we have that $u(t, \xi) \in \mathbb{D}^{\infty}$. It remains to prove that $\mathbb{E}(\|D u(t, \xi)\|)^{-p}<+\infty$ for every $p \geq 1$. By Nualart[14] Lemma 2.3.1, it suffices to prove that, for any $q \geq 2$, there exits $\varepsilon_{0}(q)>0$ such that, for all $\varepsilon<\varepsilon_{0}$,

$$
\mathbb{P}\left(\|D u(t, \xi)\|_{\mathcal{H}}^{2}<\varepsilon\right)<\varepsilon^{q}
$$

and this condition immediately follows from the estimate (40) above, choosing $\tilde{p}=\frac{q(2 \mathrm{H}-1)}{3-2 \mathrm{H}}$.

Notice that, as done for the above results, we can now extend this result to every $(t, x) \in[0, T] \times D$ and conclude that the density of the solution is infinitely differentiable with respect to the Lebesgue measure.

\section{Appendix A. Some supplementary lemmas}

The following lemmas are needed in the proof of Corollary 3 ,

Lemma 25. If $0 \leq a \leq d-1,0 \leq b \leq d-1$ then

$$
\int_{\partial D} \frac{\mathrm{d} y}{|x-y|^{a}|y-\xi|^{b}} \leq \begin{cases}c|x-\xi|^{d-1-a-b} & \text { if } a+b>d-1 \\ c & \text { if } a+b<d-1\end{cases}
$$

Lemma 26. Let $x \in \partial D, y \in \partial D$. The following estimate holds

$$
\left|\frac{\partial}{\partial \nu(x)} \Gamma(t, x, s, y)\right| \leq c(t-s)^{-\mu}|x-y|^{2 \mu-d}
$$

Proof. Proceeding as in the proof of [10, Theorem 5.2.1], we write

$$
\begin{aligned}
\frac{\partial}{\partial \nu(x)} \Gamma(t, x, s, y) & =\frac{\partial}{\partial \nu(x)} Z(t, x, s, y) \\
& =-\frac{(2 \pi)^{-\frac{d}{2}}}{2}(t-s)^{-\frac{d}{2}-1} \exp \left(-\frac{|x-y|^{2}}{2(t-s)}\right)|x-y| \cos \left(N_{x}, \overrightarrow{x y}\right)
\end{aligned}
$$

where with $N_{x}$ we denote the normal vector in $x$ and $\overrightarrow{x y}$ denotes the vector which connects $x$ with $y$. Since

$$
\left|\cos \left(N_{n}, \overrightarrow{x y}\right)\right| \leq C|x-y|
$$

we get

$$
\begin{aligned}
\left|\frac{\partial}{\partial \nu(x)} Z(t, x, s, y)\right| & \leq C(t-s)^{-\frac{d}{2}-1} \exp \left(-\frac{|x-y|^{2}}{2(t-s)}\right)|x-y|^{2} \\
& \leq C(t-s)^{-\lambda}|x-y|^{-d+2 \lambda}
\end{aligned}
$$

where in the last inequality we have used the following analytic inequality: for any $\alpha>0$ and $x>0$ it holds

$$
x^{\alpha} e^{-x} \leq \alpha^{\alpha} e^{-\alpha}
$$

and so we obtain that for any $\mu>0$ and $n \geq 1$ there exists a constant $K=K(n, \mu)$ such that for any $r>0$ :

$$
t^{-n / 2} e^{-k r^{2} / t} \leq K t^{-\lambda} r^{-n+2 \lambda}
$$

The following result is needed in order to prove the Malliavin regularity of the solution. 
Lemma 27. Under the Hypothesis (a1'), it holds

$$
\int_{S}\left(\int_{\partial D}|x-\bar{y}|^{2 \mu-d}|\alpha(\sigma, \bar{y})| \mathrm{d} \bar{y}\right)^{2} \mu(\mathrm{d} \sigma)<C\|\alpha\|_{L^{\theta}\left(\partial D ; L^{2}(S)\right)}^{2} .
$$

Proof. Using the Hölder inequality with $\theta$ satisfying Hypothesis (a1'), we get

$$
\begin{aligned}
& \int_{S}\left(\int_{\partial D}|\xi-\bar{y}|^{2 \mu-d}\right.\alpha(\sigma, \bar{y}) \mathrm{d} \bar{y})^{2} \mu(\mathrm{d} \sigma) \\
& \leq \int_{S}\left(\int_{\partial D}|\xi-\bar{y}|^{\theta^{\prime}(2 \mu-d)} \mathrm{d} \bar{y}\right)^{\frac{2}{\theta^{\prime}}}\left(\int_{\partial D}|\alpha(\sigma, \bar{y})|^{\theta} \mathrm{d} \bar{y}\right)^{\frac{2}{\theta}} \mu(\mathrm{d} \sigma) \\
& \leq C \int_{S}\left(\int_{\partial D}|\alpha(\sigma, \bar{y})|^{\theta} \mathrm{d} \bar{y}\right)^{\frac{2}{\theta}} \mu(\mathrm{d} \sigma)=C\|\alpha\|_{L^{\theta}\left(\partial D ; L^{2}(S)\right)}^{2}
\end{aligned}
$$

Notice that the condition imposed on $\theta$ is needed in order to apply Lemma 25] and so have the boundedness of the integral

$$
\int_{\partial D}|\xi-\bar{y}|^{\theta^{\prime}(2 \mu-d)} \mathrm{d} \bar{y}
$$

The following two lemmas are necessary for the proof of Theorem 23.

Lemma 28. For every $\xi \in \partial D$ and $t \in[0, T]$

$$
\mathbb{E}\|D G(t, \xi)\|_{\mathcal{H}_{\delta}}^{p} \leq C_{L, p} \delta^{p},
$$

for every $p \geq 2$, where

$$
G(t, \xi):=\int_{0}^{t} \int_{\partial D} p_{N}(t-s, \xi, \bar{y}) g(u(s, \bar{y})) \mathrm{d} \bar{y} \mathrm{~d} s .
$$

Proof. We have that

$$
\mathbf{1}_{(t-\delta, t)}(s) D_{r, \sigma} G(t, \xi)=\mathbf{1}_{(t-\delta, t)}(s) \int_{0}^{t} \int_{\partial D} p_{N}(t-s, \xi, \bar{y}) \partial_{u} g(u(s, \bar{y})) D_{r, \sigma} u(s, \bar{y}) \mathrm{d} \bar{y} \mathrm{~d} s
$$

and since $D_{r, \sigma} u(t, \xi)=0$ for every $t<r$, we get

$$
=\mathbf{1}_{(t-\delta, t)}(s) \int_{t-\delta}^{t} \int_{\partial D} p_{N}(t-s, \xi, \bar{y}) \partial_{u} g(u(s, \bar{y})) D_{r, \sigma} u(s, \bar{y}) \mathrm{d} \bar{y} \mathrm{~d} s
$$

Using Hölder inequality and the estimates on the kernel we get

$$
\begin{aligned}
& \mathbb{E}\|D G(t, \xi)\|_{\mathcal{H}_{\delta}}^{p} \leq L^{p} \mathbb{E}\left(\int_{t-\delta}^{t} \int_{\partial D} p_{N}(t-s, \xi, \bar{y})\left\|D_{r, \sigma} u(s, \bar{y})\right\|_{\mathcal{H}} \mathrm{d} \bar{y} \mathrm{~d} s\right)^{p} \\
& \leq L^{p}\left(\int_{t-\delta}^{t} \int_{\partial D} p_{N}(t-s, \xi, \bar{y})^{q} \mathrm{~d} \bar{y} \mathrm{~d} s\right)^{\frac{p}{q}}\left(\int_{t-\delta}^{t} \int_{\partial D} \mathbb{E}\left\|D_{r, \sigma} u(s, \bar{y})\right\|_{\mathcal{H}}^{p} \mathrm{~d} \bar{y} \mathrm{~d} s\right)
\end{aligned}
$$

The term $\mathbb{E}\left\|D_{r, \sigma} u(s, \bar{y})\right\|_{\mathcal{H}}^{p}$ is finite thanks to Theorem 19, so we obtain

$$
\mathbb{E}\|D G(t, \xi)\|_{\mathcal{H}_{\delta}}^{p} \leq L^{p}\left(\int_{t-\delta}^{t} \int_{\partial D} p_{N}(t-s, \xi, \bar{y}) \mathrm{d} \bar{y} \mathrm{~d} s\right)^{p},
$$


and using (11) it follows that

$$
\begin{aligned}
\left(\int_{t-\delta}^{t} \int_{\partial D} p_{N}(t-s, \xi, \bar{y})^{q} \mathrm{~d} \bar{y} \mathrm{~d} s\right)^{p} & \leq\left(\int_{\partial D}|\xi-\bar{y}|^{(2 \mu-d)} \mathrm{d} \bar{y}\right)^{p}\left(\int_{t-\delta}^{t}(t-s)^{-\mu} \mathrm{d} s\right)^{p} \\
& \leq C \delta^{p(1-\mu)} \leq C \delta^{p}
\end{aligned}
$$

and the proof is complete.

Next lemma is concerned with the norm of the Malliavin derivative $D Z(t, x)$ of the stochastic convolution process $Z(t, x)$ in the space $\mathcal{H}_{\delta}$ and is a refinement of the results in Lemma 15 .

Lemma 29. Given $\delta_{0}>0$, for every $\delta<\delta_{0}$ and every $x \in D$ and $\left.\left.t \in\right] 0, T\right]$,

$$
\|D Z(t, x)\|_{\mathcal{H}_{\delta}}^{2} \geq C_{\delta_{0}, T, \alpha_{0}} \delta^{2 H-1} .
$$

Proof. Proceeding as in the proof of Lemma 15, we get

$$
\begin{aligned}
\|D Z(t, x)\|_{\mathcal{H}_{\delta}}^{2}= & \int_{t-\delta}^{t} \int_{t-\delta}^{t} \int_{S} D_{s, \sigma} Z(t, x) D_{r, \sigma} Z(t, x)|s-r|^{2 H-2} \mu(\mathrm{d} \sigma) \mathrm{d} r \mathrm{~d} s \\
& \geq C \delta^{d-1} \int_{t-\delta}^{t} \int_{t-\delta}^{t}(t-s)^{-\frac{d}{2}}(t-r)^{-\frac{d}{2}}|s-r|^{2 H-2} \exp \left(-\frac{\delta^{2}}{t-s}\right) \exp \left(-\frac{\delta^{2}}{t-r}\right) \mathrm{d} r \mathrm{~d} s \\
& =C \delta^{d-1} \int_{0}^{\delta} \int_{0}^{\delta} \sigma^{-\frac{d}{2}} \rho^{-\frac{d}{2}}|\rho-\sigma|^{2 H-2} \exp \left(-\frac{\delta^{2}}{\sigma}\right) \exp \left(-\frac{\delta^{2}}{\rho}\right) \mathrm{d} \sigma \mathrm{d} \rho \\
& \geq C \delta^{d-1} e^{-2 \delta} \int_{0}^{\delta} \int_{0}^{\delta} \sigma^{-\frac{d}{2}} \rho^{-\frac{d}{2}}|\rho-\sigma|^{2 H-2} \mathrm{~d} \sigma \mathrm{d} \rho \\
& \geq C \delta^{-1} e^{-2 \delta} \int_{0}^{\delta} \int_{0}^{\delta}|\rho-\sigma|^{2 H-2} \mathrm{~d} \sigma \mathrm{d} \rho \geq C \delta^{-1} e^{-2 \delta} \frac{\delta^{2 H}}{2 H(2 H-1)}=C_{\delta_{0}, T, \alpha_{0}} \delta^{2 H-1}
\end{aligned}
$$

Lemma 30. Let $\left\{V_{n}, n \geq 0\right\}$ be a sequence of processes in $L_{W}^{p}\left(0, T \times \partial D ; \mathcal{H}^{\otimes k}\right), p \geq 2, k \geq 0$ (in the case $k=0$ we mean $\mathcal{H}=\mathbb{R}$ ) defined recursively by

$$
V_{n+1}(t, x)=V_{0}(t, x)+\int_{r}^{t} \int_{\partial D} p_{N}(t-s, x, \bar{y}) h_{n}(s, \bar{y}) V_{n}(s, \bar{y}) \mathrm{d} \bar{y} \mathrm{~d} s
$$

and $V_{0}$ is given. Moreover, $h_{n}$ is a sequence of equi-bounded real-valued random processes.

Then there exists a suitable $\lambda$ in the definition of the norm $\|\cdot\|_{L_{W}^{p}}$ (see formula (24)) and a constant $C<1$ such that the following estimate holds (compare for instance with (26)):

$$
\left\|V_{n+1}-V_{n}\right\|_{L_{W}^{p}} \leq C\left\|V_{n}-V_{n-1}\right\|_{L_{W}^{p}}
$$

Proof. In order to prove (46), let us consider in the space $L_{W}^{p}\left([0, T] \times \partial D ; \mathcal{H}^{\otimes k}\right)$ the equivalent norm

$$
\|f\|_{L_{W}^{p}}=\mathbb{E} \int_{0}^{T} e^{-\lambda t}\|f(t)\|_{L^{p}(\partial D ; \mathcal{H} \otimes k)}^{p} \mathrm{~d} t,
$$

for some constant $\lambda$ to be chosen later. The norm is equivalent to the standard one. 
Setting for simplicity $\tilde{V}(t, x):=\left(V_{n}-V_{n-1}\right)(t, x)$ and using Hölder inequality and the assumption of equi-boundedness on $h, \sup h(t, \bar{x}) \leq L<\infty \mathbb{P}$-a.s., we have that

$$
\begin{aligned}
\mathbb{E}\left\|\left(V_{n+1}-V_{n}\right)(t, x)\right\|_{\mathcal{H}^{\otimes k}}^{p} & \left.=\mathbb{E} \| \int_{r}^{t} \int_{\partial D} p_{N}(t-s, x, \bar{y}) h(s, \bar{y})\right)\left(V_{n}-V_{n-1}\right)(s, \bar{y}) \mathrm{d} \bar{y} \mathrm{~d} s \|_{\mathcal{H}^{\otimes k}}^{p} \\
\leq & L \mathbb{E}\left(\int_{r}^{t} \int_{\partial D} p_{N}(t-s, x, \bar{y})\|\tilde{V}(s, \bar{y})\|_{\mathcal{H} \otimes k} \mathrm{~d} \bar{y} \mathrm{~d} s\right)^{p} \\
\leq & L\left(\int_{0}^{t} \int_{\partial D} p_{N}(t-s, x, \bar{y}) e^{\lambda s(q-1)} \mathrm{d} \bar{y} \mathrm{~d} s\right)^{\frac{p}{q}} \times \\
& \times \mathbb{E}\left(\int_{0}^{t} \int_{\partial D} p_{N}(t-s, x, \bar{y}) e^{-\lambda s}\|\tilde{V}(s, \bar{y})\|_{\mathcal{H} \otimes k} \mathrm{~d} \bar{y} \mathrm{~d} s\right) .
\end{aligned}
$$

Using estimate (11) we obtain the following

$$
\begin{aligned}
\left(\int_{0}^{t} \int_{\partial D} p_{N}(t-s, x, \bar{y}) e^{\lambda s(q-1)} \mathrm{d} \bar{y} \mathrm{~d} s\right)^{\frac{p}{q}} & =\left(\int_{0}^{t} \int_{\partial D} p_{N}(s, x, \bar{y}) e^{\frac{\lambda(t-s)}{p-1}} \mathrm{~d} \bar{y} \mathrm{~d} s\right)^{p-1} \\
& \leq\left(\int_{\partial D}|x-\bar{y}|^{2 \mu-d} \mathrm{~d} \bar{y} \int_{0}^{t} s^{-\mu} e^{\frac{\lambda(t-s)}{p-1}} \mathrm{~d} s\right)^{p-1} \\
& \leq C \frac{1}{\lambda^{(1-\mu) /(p-1)}} e^{\lambda t}
\end{aligned}
$$

where $C=C(p, \mu)$ is uniformly bounded for fixed $\mu$ and $p \geq 2$. So, by means of the Fubini's Theorem, we get

$$
\begin{aligned}
\left\|V_{n+1}-V_{n}\right\|_{L_{W}^{p}}^{p} & =\int_{r}^{T} \int_{\partial D} \mathbb{E}\left\|V_{n+1}-V_{n}\right\|_{\mathcal{H}^{\otimes k}}^{p} \mathrm{~d} x e^{-\lambda t} \mathrm{~d} t \\
& \leq C \frac{1}{\lambda^{(1-\mu) /(p-1)}} \int_{r}^{T} \int_{\partial D} e^{\lambda t} \int_{r}^{t} \int_{\partial D} p_{N}(t-s, x, \bar{y}) e^{-\lambda s} \mathbb{E}\|\tilde{V}(s, \bar{y})\|_{\mathcal{H} \otimes k}^{p} \mathrm{~d} \bar{y} \mathrm{~d} s \mathrm{~d} x e^{-\lambda t} \mathrm{~d} t \\
& =C \frac{1}{\lambda^{(1-\mu) /(p-1)}} \int_{r}^{T} \int_{\partial D} \mathbb{E}\|\tilde{V}(s, \bar{y})\|_{\mathcal{H}^{\otimes k}}^{p} e^{-\lambda s}\left(\int_{s}^{T} \int_{\partial D} p_{N}(t-s, \xi, \bar{y}) \mathrm{d} x \mathrm{~d} t\right) \mathrm{d} \bar{y} \mathrm{~d} s \\
& \leq C \frac{1}{\lambda^{(1-\mu) /(p-1)}} \int_{r}^{T} \int_{\partial D} \mathbb{E}\|\tilde{V}(s, \bar{y})\|_{\mathcal{H}^{\otimes k}}^{p} e^{-\lambda s} \mathrm{~d} \bar{y} \mathrm{~d} s \\
& \leq C \lambda^{\frac{\mu-1}{p-1}}\left\|V_{n}-V_{n-1}\right\|_{L_{W}^{p}}^{p},
\end{aligned}
$$

where, thanks again to Lemma 25 (recall that $\frac{1}{2}<\mu<1$ and so $2 \mu-d<d-1$ ),

$$
\begin{aligned}
\int_{s}^{T} \int_{\partial D} p_{N}(t-s, \xi, \bar{y}) \mathrm{d} \xi \mathrm{d} t & \leq \int_{s}^{T} \int_{\partial D}|\xi-\bar{y}|^{2 \mu-d}(t-s)^{-\mu} \mathrm{d} \xi \mathrm{d} t \\
& \leq \int_{0}^{T} t^{-\mu} \mathrm{d} t \int_{\partial D}|\xi-\bar{y}|^{2 \mu-d} \mathrm{~d} \xi \leq C_{\mu} T^{1-\mu}=C_{\mu, T}
\end{aligned}
$$

Then we see that there exists $\lambda$ large enough such that $C \lambda^{\frac{\mu-1}{p-1}} \leq 1-\varepsilon<1$ and this proves the Lemma. $\square$ 


\section{REFERENCES}

[1] V. Barbu, S. Bonaccorsi, and L. Tubaro. A stochastic parabolic equation with nonlinear flux on the boundary driven by a Gaussian noise. SIAM J. Math. Anal., 46(1):780-802, 2014.

[2] V. Barbu, S. Bonaccorsi, and L. Tubaro. Stochastic parabolic equations with nonlinear dynamical boundary conditions. J. Math. Anal. Appl., 427(1):484-498, 2015.

[3] S. Bonaccorsi. Volterra equations perturbed by a Gaussian noise. In Seminar on Stochastic Analysis, Random Fields and Applications V, volume 59 of Progr. Probab., pages 37-55. Birkhäuser, Basel, 2008.

[4] S. Bonaccorsi and D. Mugnolo. Existence of strong solutions for neuronal network dynamics driven by fractional Brownian motions. Stochastics and Dynamics, 10(3):441-464, 2010.

[5] S. Bonaccorsi and C. A. Tudor. Dissipative stochastic evolution equations driven by general Gaussian and nonGaussian noise. J. Dynam. Differential Equations, 23(4):791-816, 2011.

[6] S. Bonaccorsi and M. Zanella. Existence and regularity of the density for solutions of stochastic differential equations with boundary noise. Infin. Dimens. Anal. Quantum Probab. Relat. Top., 19(1):1650007, 24, 2016.

[7] G. Da Prato and J. Zabczyk. Stochastic equations in infinite dimensions, volume 44 of Encyclopedia of Mathematics and its Applications. Cambridge University Press, Cambridge, 1992.

[8] T. E. Duncan, B. Maslowski, and B. Pasik-Duncan. Stochastic equations in Hilbert space with a multiplicative fractional Gaussian noise. Stochastic Process. Appl., 115(8):1357-1383, 2005.

[9] T. E. Duncan, B. Pasik-Duncan, and B. Maslowski. Fractional Brownian motion and stochastic equations in Hilbert spaces. Stoch. Dyn., 2(2):225-250, 2002.

[10] A. Friedman. Partial differential equations of parabolic type. Prentice-Hall Inc., Englewood Cliffs, N.J., 1964.

[11] J.-L. Lions and E. Magenes. Non-homogeneous boundary value problems and applications. Vol. I. Springer-Verlag, New York, 1972.

[12] X. Mao and L. Markus. Wave equation with stochastic boundary values. J. Math. Anal. Appl., 177(2):315-341, 1993.

[13] B. Maslowski and D. Nualart. Evolution equations driven by a fractional Brownian motion. J. Funct. Anal., 202(1):277-305, 2003.

[14] D. Nualart. The Malliavin calculus and related topics. Probability and its Applications (New York). Springer-Verlag, Berlin, second edition, 2006.

[15] R. B. Sowers. Multidimensional reaction-diffusion equations with white noise boundary perturbations. Ann. Probab., 22(4):2071-2121, 1994.

[16] J. B. Walsh. An introduction to stochastic partial differential equations. In École d'été de probabilités de Saint-Flour, XIV-1984, volume 1180 of Lecture Notes in Math., pages 265-439. Springer, Berlin, 1986.

[17] X. Yang and T. Zhang. Estimates of heat kernels with Neumann boundary conditions. Potential Anal., 38(2):549$572,2013$.

Stefano BonacCORsi

Dipartimento di Matematica, Università di Trento, via Sommarive 14, 38123 Povo (Trento), Italia

E-mail address: stefano.bonaccorsi@unitn.it

MARGHERITA ZANELLA

Dipartimento di Matematica, Università di Pavia, via Ferrata 5, 27100 Pavia, Italia

E-mail address: margherita.zanella01@ateneopv.it 